\title{
Theoretical study of metal borides stability
}

\author{
Aleksey N. Kolmogorov and Stefano Curtarolo \\ Department of Mechanical Engineering and Materials Science, Duke University, Durham, NC 27708
}

(Dated: February 6, 2008)

\begin{abstract}
We have recently identified metal-sandwich (MS) crystal structures and shown with ab initio calculations that the MS lithium monoboride phases are favored over the known stoichiometric ones under hydrostatic pressure [Phys. Rev. B 73, 180501(R) (2006)]. According to previous studies synthesized lithium monoboride tends to be boron-deficient, however the mechanism leading to this phenomenon is not fully understood. We propose a simple model that explains the experimentally observed off-stoichiometry and show that compared to such boron-deficient phases the MS-LiB compounds still have lower formation enthalpy under high pressures. We also investigate stability of MS phases for a large class of metal borides. Our $a b$ initio results suggest that MS noble metal borides are less unstable than the corresponding $\mathrm{AlB}_{2}$-type phases but not stable enough to form under equilibrium conditions.
\end{abstract}

\section{INTRODUCTION}

The interest in the $\mathrm{AlB}_{2}$ family of metal diborides re-emerged after the discovery of superconductivity in $\mathrm{MgB}_{2}$ with a surprisingly high transition temperature of $39 \mathrm{~K}[1]$. Boron $p$-states have been shown to be key for both stability and superconductivity in these compounds 2, 3, 4]. $\mathrm{MgB}_{2}$ is a unique metal diboride because it has a significant density of boron $p \sigma$-states at the Fermi level which give rise to the high $\mathrm{T}_{c}$ superconductivity, and yet enough of them are filled for the compound to be structurally stable 2, 3, 4]. The effectively hole-doped noble- and alkali-metal diborides would have higher $p \sigma$ density of states (DOS) at $\mathrm{E}_{F}$, but they have been demonstrated to be unstable under normal conditions 4]. The effort to achieve higher $\mathrm{T}_{c}$ has thus primarily focused on doping magnesium diboride with various metals; however, doping this material has proven to be difficult [5] and no improvement on $\mathrm{T}_{c}$ has yet been reported. According to a recent theoretical study of nonlocal screening effects in metals, $\mathrm{MgB}_{2}$ may already be optimally doped $[6]$. Lithium borocarbide with a doubled $\mathrm{AlB}_{2}$ unit cell has been suggested as a possible high- $\mathrm{T}_{c}$ superconductor under hole-doping [7], but disorder in the heavily doped $\mathrm{Li}_{x} \mathrm{BC}$ appears to forbid superconductivity above $2 \mathrm{~K}[\mathrm{8}]$.

In this work we investigate whether there could be stable high- $\mathrm{T}_{c}$ superconducting metal borides in configurations beyond the standard $\mathrm{AlB}_{2}$ prototype. We have recently proposed metal-sandwich (MS) structures MS1 and MS2, which also have $s p^{2}$ layers of boron but separated by two metal layers [9]. Despite their rather simple unit cells these structures have apparently never been considered before. As we demonstrate below, identification of the MS structures is not straightforward because they represent a local minimum not usually explored with current compound prediction strategies [9]. We reveal trends in the cohesion of MS phases by calculating formation energies for a large class of metal borides and show that some monovalent-metal borides benefit from having additional layers of metal. The MS noblemetal borides still have positive formation energy, but they are less unstable than the $\mathrm{AlB}_{2}$-type phases. This result helps resolve the question of what phases would form first in the noble-metal boride systems under nonequilibrium conditions $10,11,12,13$.

Our main finding concerns the Li-B system, in which the MS lithium monoboride is stable enough to compete against the known stoichiometric phases. According to our previous ab initio calculations the MS lithium monoboride is comparable in energy to these phases under normal conditions, but it becomes the ground state at $50 \%$ concentration under moderate hydrostatic pressures 9 . Here we extend the analysis to non-stoichiometric Li-B phases which could potentially intervene in the synthesis of the MS phases. In particular, synthesized lithium monoboride with linear chains of boron is known to be boron-deficient for reasons not fully understood so far. We simulate the incommensurate $\mathrm{LiB}_{y}$ phases (notation explained in Ref. [14]) by constructing a series of small periodic $\mathrm{Li}_{2 n} \mathrm{~B}_{m}$ structures and show that the minimum formation energy is achieved for $y \approx 0.9$, in very good agreement with the experimentally observed values. Using this simple model of the off-stoichiometry phases with linear chains of boron we demonstrate that relative to them MS-LiB still has lower formation enthalpy under high pressures. Simulations of other alkali-metal borides, $\mathrm{MB}_{y}(\mathrm{M}=\mathrm{Na}, \mathrm{K}, \mathrm{Rb}, \mathrm{Cs})$, suggest that that these nearly stoichiometric phases might form under moderate pressures.

The paper is divided in the sections describing: 2) simulation details; 3 ) construction of the MS prototypes; 4) stability of MS phases for a large class of metal borides; 5) detailed investigation of the Li-B system; 6) simulations of other monovalent and higher-valent metal borides; 7) summary of the electronic and structural properties of the MS phases. 


\section{COMPUTATION DETAILS}

Present $a b$ initio calculations are performed with Vienna Ab-Initio Simulation Package VASP [15, 16] with Projector Augmented Waves (PAW) [17] and exchangecorrelation functionals as parametrized by Perdew, Burke, and Ernzerhof (PBE) 18] for the Generalized Gradient Approximation (GGA). Because of a significant charge transfer between metal and boron in most structures considered we use PAW pseudopotentials in which semi-core states are treated as valence. This is especially important for the Li-B system as discussed in Refs. [9, 19]. Simulations are carried out at zero temperature and without zero-point motion; spin polarization is used only for magnetic alloys. We use an energy cutoff of $398 \mathrm{eV}$ and at least 8000/(number of atoms in unit cell) $\mathbf{k}$-points distributed on a Monkhorst-Pack mesh [20]. We also employ an augmented plane-wave+local orbitals (APW+lo) code WIEN2K to plot characters of electronic bands 21]. All structures are fully relaxed. Our careful tests show that the relative energies are numerically converged to within $1 \sim 2 \mathrm{meV} /$ atom.

Construction of binary phase diagrams $\mathrm{A}_{x} \mathrm{~B}_{1-x}$ is based on the calculated formation enthalpy $H_{f}$, which is determined with respect to the most stable structures of pure elements. For boron there are two competing phases $\alpha$-B and $\beta$-B 22]; we use $\alpha$-B (Ref. [4]), theoretically shown to be the more stable phase at low temperatures and high pressures [22]. A structure at a given composition $x$ is considered stable (at zero temperature and without zero-point motion) if it has the lowest formation enthalpy for any structure at this composition and if on the binary phase diagram $H_{f}(x)$ it lies below a tie-line connecting the two stable structures closest in composition to $x$ on each side.

\section{IDENTIFICATION OF MS PROTOTYPES}

Data-mining of quantum calculations (DMQC), introduced in our previous work 23], is a theoretical method to predict the structure of materials through efficient re-use of $a b$ initio results. The DMQC iteratively determines correlations in the calculated energies on a chosen library of binary alloys and structure types. The last work has demonstrated that for a set of 114 crystal structures and 55 binary metallic alloys the method gives an almost perfect prediction of the ground states (within the library) in a fraction of all possible computations [23, 24]. The speed-up (commonly by a factor from 3 to 4 ) is achieved by the method's rational strategy for suggesting the next phase to be evaluated. An essential feature of these calculations is the full relaxation of the considered structures, which ensures an accurate determination of the correlations in the chosen library 25].

We have recently begun expanding the $114 \times 55$ library

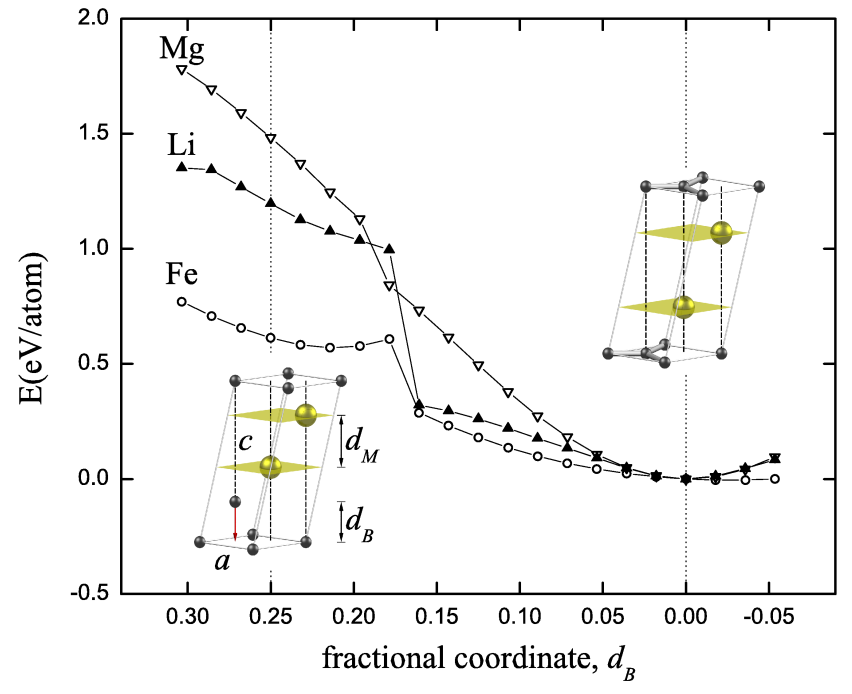

FIG. 1: (color online). Energy barrier as a function of boron fractional coordinate $d_{B}$ for a transition path from V2 $\left(d_{B} \approx 0.25[26]\right)$ to $\operatorname{MS} 1\left(d_{B} \approx 0\right.$, see text) for different metal borides. For each value of $d_{B}$ the energy is minimized by adjustment of the remaining free parameters $a, c$, and the metal fractional coordinate $d_{M}$.

of $a b$ initio energies of binary alloys 23 to include metal borides. Boron tends to form covalent bonds in intermetallic compounds; to have this correlation in future predictions with the DMQC we needed first to add a few compound-forming metal-boride systems into the library. Introduction of a new system involves calculations of energies for all the prototype entries in the library. Surprisingly, in the very first system considered, $\mathrm{Mg}-\mathrm{B}$, one of the fcc structures with 4-atom unit cell at 50\% concentration, $\mathrm{A}_{2} \mathrm{~B}_{2}$ fcc-(111) (or V2 [26]), relaxed almost all the way down to the $\mathrm{AlB}_{2}-\mathrm{MgB}_{2} \leftrightarrow$ hcp-Mg tie-line. Significant relaxations are not uncommon in our simulations; they usually correspond to the transformation from a starting configuration to a known stable prototype and are automatically detected by the change in the symmetry. The magnesium monoboride phase, however, retained its original space group R $\overline{3} \mathrm{~m}$ (\#166).

Having examined the relaxation process we found that there is a continuous symmetry-conserving path from V2 to a new structure MS1 9, 27]. V2 has 4 atoms per unit cell with 4 free parameters $a, c, d_{B}$, and $d_{M}$ (the last two are fractional distances between boron and metal layers), so that atoms are constrained only to the vertical lines (see Fig. 1). The perfect fcc lattice corresponds to $c / a=4 \sqrt{2 / 3}$ and $d_{B}=d_{M}=0.25$, but this special case does not grant additional symmetry operations and local relaxation have been seen in some metallic systems [26]. 
In metal borides a more dramatic transformation leads to a much more stable configuration: boron atoms rearrange themselves to form covalent bonds in a hexagonal layer $\left(d_{B} \rightarrow 0\right)$ rather then share electrons in closepacked triangular layers, while metal atoms remain in a close-packed bilayer. We have checked other alkali, alkaline and transition metal borides not present in the DMQC library and confirmed that they all benefit from this transformation; however, some electron-rich systems might not escape from the local fcc-type minimum, as shown in Fig. 1 for FeB. This could be a reason why the MS1 prototype has apparently been overlooked so far. We would like to point out that identification of new prototypes is not an intended function of the DMQC. This interesting accidental result should be credited to the exhaustive consideration of all candidates (regardless of how unlikely they seem to be a stable phase - an fcc supercell is hardly a suitable configuration for a magnesium boride phase) and the careful structural relaxation in the calculation of their ground state energies.

We proceed by constructing a library of related MS prototypes. Structures, where the metal atoms closest to boron sit directly above the center of boron hexagons, are uniquely specified by the positions of the metal layers (such as $\alpha, \beta, \gamma$, in Fig. 2). The MS1 structure can thus also be labeled as $|\alpha \beta|$ : the Greek letters show the positions of the two metal layers and vertical bars correspond to boron layers. A hexagonal supercell for this phase 27] is obtained when the last metal layer matches the first: $|\alpha \beta| \beta \gamma|\gamma \alpha|$. The fourth metal layer can alternatively be shifted back to site $\alpha$ (see Fig. 2(d)), resulting in another structure at the same stoichiometry MS2 $(|\alpha \beta| \beta \alpha \mid)$ 9, 28]. Examples of more metal-rich structures are $|\alpha \beta \alpha|$ (MS3) 29] and $|\alpha \beta \gamma|$. Various stoichiometries can also be achieved by combination of smaller cells, i.e. $|\alpha \beta| \beta \mid$ (MS4) and $|\alpha| \alpha \beta|\beta \alpha|$. In this notation the $\mathrm{AlB}_{2}$-prototype is labeled simply as $|\alpha|$, which we will use henceforth to avoid confusion with the aluminum diboride compound. Positioning metal atoms above boron hexagon centers is not the only possibility. Stacking faults have been experimentally observed in $\mathrm{MgB}_{2}$ 30], though such defects have been shown to be energetically costly for this compound 31]. We have constructed a periodic structure $\left(\delta-\mathrm{MB}_{2}\right)$ with 3 atoms per unit cell where the metal atoms in $|\alpha|$ are shifted along $(\mathbf{a}+\mathbf{b})$ to be above the middle of a boron-boron bond [32].

\section{STABILITY OF THE MS PHASES}

Formation of a particular compound in systems with a few competing phases is determined by a number of factors, i.e. the ground state energy, synthesis conditions, thermodynamic and kinetic effects. Comparison of highthroughput ab initio results 24, 33, 34] with experimental databases has shown that the calculated total energies

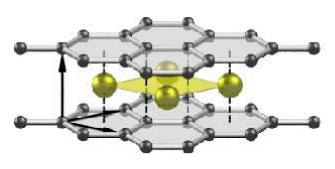

a) $\operatorname{AlB}_{2}(|\alpha|)$

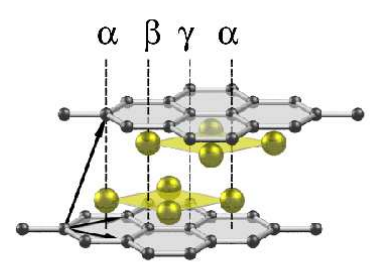

c) $\operatorname{MS1}(|\alpha \beta|)$

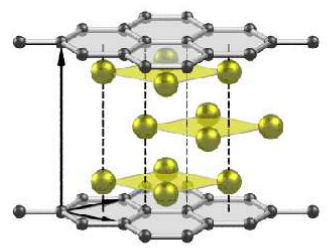

e) $\operatorname{MS} 3(|\alpha \beta \alpha|)$

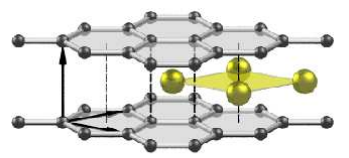

b) $\delta$

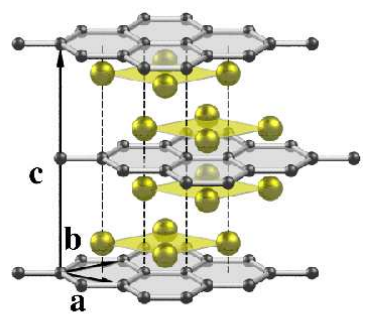

d) $\operatorname{MS} 2(|\alpha \beta| \beta \alpha \mid)$

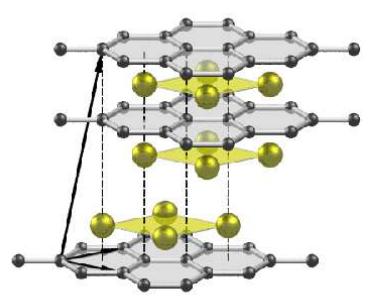

f) MS4 ( $|\alpha \beta| \beta \mid)$
FIG. 2: (color online). Known $\mathrm{AlB}_{2}$ and proposed MS structures for metal borides. The hexagonal layers of boron (grey) are separated by triangular layers of metal (yellow, only four atoms per layer are shown). Except for the $\delta$ structure shown in b) 32], all structures have metal layers positioned above the middle of the nearest boron hexagons. The alternative notations given in brackets are explained in the text. The concentration of metal in these structures is $33 \%\left(\mathrm{AlB}_{2}\right), 33 \%$ $(\delta), 50 \%$ (MS1), 50\% (MS2), 60\% (MS3), and $43 \%$ (MS4).

alone allow to identify the correct phases observed in the experiment in about $96.7 \%$ of investigated cases (Eq. 3 in Ref. 33]). In this section we use the total energy criterium to narrow down the set of systems in which the MS phases might occur.

We first calculate the formation energy for a large library of alkali, alkaline, and transition metals in the $|\alpha|$ and MS1 configurations (Fig. [3). Our results for $|\alpha|-\mathrm{MB}_{2}$ are consistent with the previous calculation by Oguchi [4] (note that we put noble metals in the first valence group). The MS1 phases exhibit a similar trend in cohesion: they are most stable for tetra-valent metal borides. It is convenient to analyze the stability of the MS phases by comparing them to the corresponding $|\alpha|$-phases because the MS structures are effectively a combination of the $|\alpha|-$ structure and additional layers of metal. Three immediate effects can be expected from the insertion of an extra metal layer: 1) different strain conditions between the boron network and the triangular layers of metal; 2) 


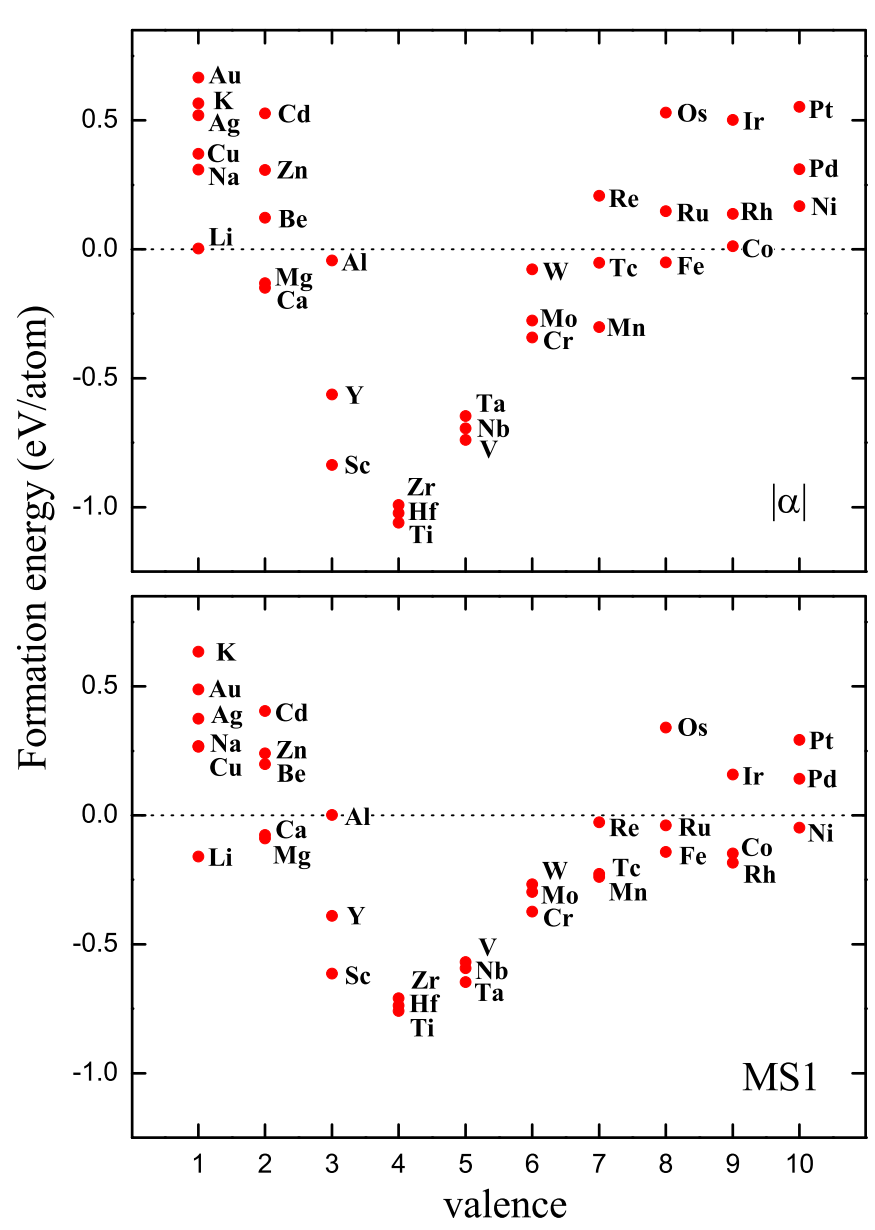

FIG. 3: (color online). Calculated formation energy for $|\alpha|$ (top) and MS1 (bottom) metal borides. The results for $|\alpha|-$ $\mathrm{MB}_{2}$ are consistent with the previous study [4] (mind the minus sign difference between formation energy and heat of formation).

different doping level of the boron layer; 3) significant reduction of interlayer overlaps between $p$-orbitals of boron due to the increase in the interlayer distance. To decouple these effects we calculate relative stability of the MS1 phase with respect to phase separation into $|\alpha|$ and pure element for the large library of metal borides. The relative stability for compound $\mathrm{M}_{x} \mathrm{~B}_{1-x}(\equiv X)$ is defined as

$$
\Delta E_{X} \equiv E_{X}-\frac{3}{2}\left[(1-x) E_{|\alpha|}+\left(x-\frac{1}{3}\right) E_{\text {pure }}\right]
$$

(all energies are per atom), and reflects whether a metal layer prefers to be in a layered boride environment or stay in pure bulk structure. To illustrate the amount of strain in the system we plot this energy difference versus equilibrium intralayer distance in pure fcc or hcp

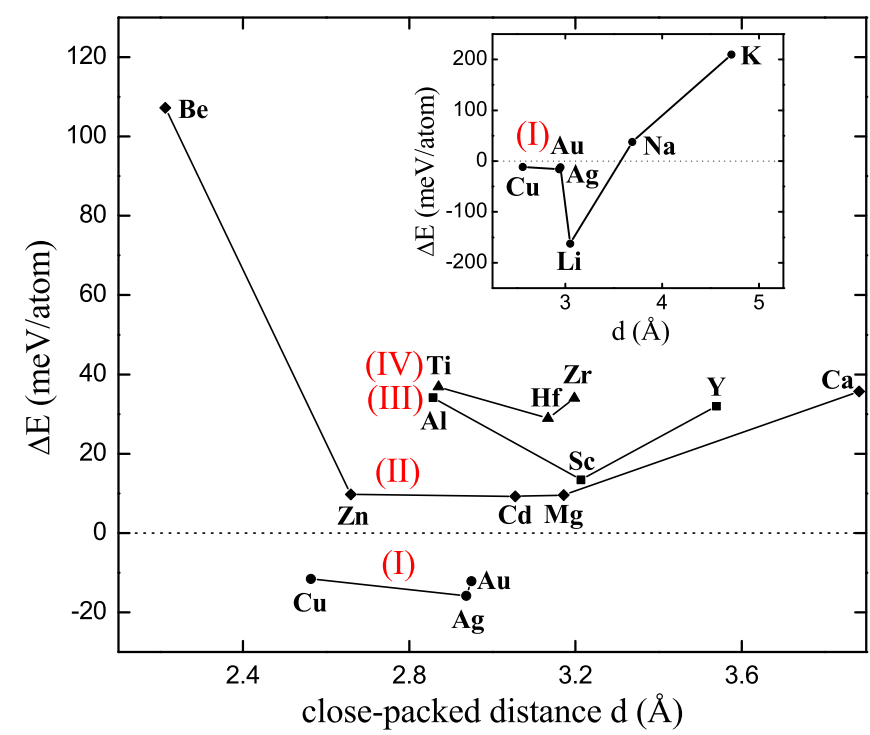

FIG. 4: (color online). Relative stability of the MS1 metal borides with respect to phase separation into $|\alpha|$ and pure metal phases (Eq. 1) as a function of the close-packed distance defined in the text. The metal borides are grouped and labeled according to the valence of metal (from 1 to 4). Inset: the same for noble- and alkali-metal borides.

bulk metal structure, whichever is more stable at zero temperature 35. Figure 4 shows that monoborides of metals in the same valence group and similar dimensions (for example $\mathrm{Zn}, \mathrm{Cd}$, and $\mathrm{Mg}$ ) have close relative stability. Metal layers mismatched with the boron layer (for example $\mathrm{Be}, \mathrm{Ca}, \mathrm{Na}$, and $\mathrm{K}$ ) cause a significant energy penalty when inserted in the respective diborides.

Another general trend captured in Fig. 4 is a consistent decrease of the MS1 phase relative stability with the increase of valence electrons (up to four). Relative stability depends on the binding mechanisms in all the three phases in Eq. 1 and the analysis of its variation with the metal valence is not straightforward. For monovalent metal borides the observed gain in binding for the MS1 phase is consistent with the fact that diborides of low-valent metals have available $p \sigma$ bonding states and stabilize as the metal valence increases 2, 44]. However, charge redistribution in these phases may follow different scenarios: in $|\alpha|$ metal atoms are exposed to boron and become almost entirely ionized 2], while in MS1 boron extracts charge through the surface of the metal bilayer and likely leaves more charge in the metal system (Section 7). With increasing valence in the transition metal series of diborides bonding $p \sigma$ and $d-p \pi$ bands become occupied, so that the binding reaches its maximum for Group IV metals and eventually goes down[4]. Conse- 
quently, we observe a noticeable increase in the relative stability of the MS1 structure for higher-valent metals (in fact, all metal diborides with at least five valence electrons benefit from insertion of an extra metal layer with the largest gain of $-340 \mathrm{meV} /$ atom obtained for $\mathrm{RhB}$ ). However, these electron-rich systems allow other phases with significantly lower energies (prototypes $\mathrm{NiAs}, \mathrm{NaCl}$, FeB-b, etc. 13]). Hence, we focus on low-electron systems that have been shown to stabilize through incorporation of extra metal layers and could compete with existing phases.

\section{LI-B SYSTEM}

Overview. A few compounds at different stoichiometries have been reported for the Li-B system 13, 36, 37, 38, 39, 40, 41]. On the boron-rich side the experimentally reproducible compounds $\mathrm{Li}_{3} \mathrm{~B}_{14}$ and $\mathrm{LiB}_{3}$ have large unit cells with fractional occupancies $13,36,37$ and cannot be presently simulated with $a b$ initio methods with desired degree of accuracy. The composition of the most lithiumrich $\mathrm{LiB}_{y}$ compounds (near $50 \%$ concentration) apparently depends on synthesis conditions and post-synthesis treatment, as the reported values for $y$ range from 0.8 to 1 (notation explained in Ref. 14]). In the early experiments the formed compounds were ascribed compositions $\mathrm{Li}_{5} \mathrm{~B}_{4}$ 42, 43] or $\mathrm{Li}_{7} \mathrm{~B}_{6}$ [44]; Wang at al. used a rhombohedral model to explain the observed x-ray patterns [42, 45]. However, a more consistent interpretation of the available x-ray data on nearly stoichiometric lithium monoboride has been recently given by Liu et al. 38. The authors demonstrated that the main x-ray peaks can be indexed with a four-atom hexagonal unit cell $\alpha-\mathrm{LiB}$ (Fig. $5(\mathrm{a})$ ), which consists of linear chains of boron embedded in hexagonal lithium shells 38 .

While the simple $\alpha$-LiB sheds light on what the structure of the lithium monoboride is, an important question remains open as to why the $\mathrm{LiB}_{y}$ compounds are boron-deficient. Wörle and Nesper have offered an insightful model of $\mathrm{LiB}_{y}$, in which the boron chains are uncorrelated and incommensurate with the lithium sublattice 46. By using a large unit cell containing 32,000 atoms the authors reproduced a kink at $2 \theta \approx$ $60^{\circ}$ in the x-ray pattern and attributed it to the average boron-boron distance of $1.59 \AA$. They also suggested that the boron chains could be dimerized or have vacancies [46]. According to a recent theoretical study, boron chains in lithium monoboride are not expected to dimerize but might indeed be able to slide freely along the lithium sublattice 39].

Model of the $\mathrm{LiB}_{y}$ compounds. Simulation of the disordered $\mathrm{LiB}_{y}$ compounds is essential for finding a possible stability region of the MS-LiB phases. While the large unit cells with thousands of atoms are needed to reproduce the x-ray data, such sizes prohibit the use of

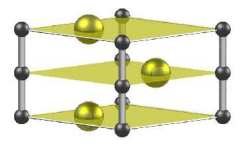

a) $\alpha-\mathrm{LiB}$

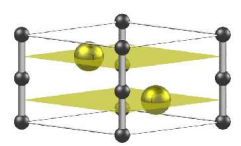

b) $\beta$-LiB

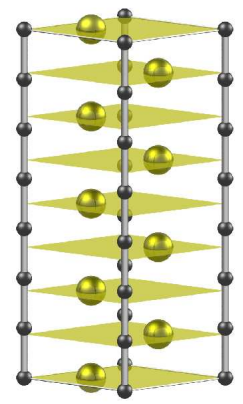

c) $\mathrm{Li}_{8} \mathrm{~B}_{7}$
FIG. 5: (color online). Structures with linearly chained boron immersed in a lithium sublattice: the known $\alpha$ - $\mathrm{LiB}$ and $\beta$-LiB have commensurate sublattices; the proposed $\mathrm{Li}_{2 n} \mathrm{~B}_{m}$ series (only $\mathrm{Li}_{8} \mathrm{~B}_{7}$ is shown) allows to simulate the incommensurate off-stoichiometry $\mathrm{LiB}_{y}$ phases.

$a b$ initio methods for ground state energy calculations. Therefore, we simulate the incommensurate $\mathrm{LiB}_{y}$ compounds by constructing a series of relatively small commensurate $\mathrm{Li}_{2 n} \mathrm{~B}_{m}$ phases. The number of lithium atoms in a unit cell must be even since they occupy alternating sites along the $c$-axis. To determine the optimal relative position of the two sublattices we fix only the $z$ components of one lithium and one boron atoms and allow all the other degrees of freedom to relax. We find that the relative placement matters only for the smallest $\mathrm{Li}_{2} \mathrm{~B}_{2}$ unit cells: as we have shown in Ref. [9] the energy difference between $\alpha$ - $\mathrm{LiB}$ and $\beta-\mathrm{LiB}$ is $10 \mathrm{meV} /$ atom. For all other periodic structures the barriers to sliding for the two sublattices are below $1 \mathrm{meV}$ per unit cell. The situation is similar to the relative motion in multiwalled carbon nanotubes, where the rigid layers interact weakly with one another: in long-period commensurate nanotubes the barriers to intertube sliding are extremely small, and in incommensurate ones the intertube sliding mode is gapless [47. Local relaxations in $\operatorname{Li}_{2 n} \mathrm{~B}_{m}(m>2$, $m \neq 2 n$ ) are insignificant due to the rigidity of the boronboron chains and a smooth charge density distribution along the chains 39$]$.

Stability and structure of the $\mathrm{LiB}_{y}$ compounds. Figure 6 shows an immediate benefit for the lithium monoboride to change composition: as the level of lithium concentration increases by a few percent the phase undergoes stabilization by over $20 \mathrm{meV} /$ atom at zero pressure. The points on the enthalpy versus concentration plot for the $\mathrm{Li}_{2 n} \mathrm{~B}_{m}$ series nicely fit to a parabola (with minimum at $y \approx 0.894$ at zero pressure). This leads to an interesting situation, in which the $\mathrm{LiB}_{y}$ phases are actually stable over a range of concentrations. The lower boron concentration limit corresponds to $y_{\min } \approx 0.874$ (the tangent to the parabola going through $x=1$, shown in Fig. 6), while the higher one depends on the location of $\mathrm{LiB}_{3}$ on the phase diagram and should be around $y_{\max } \approx 0.9$. 


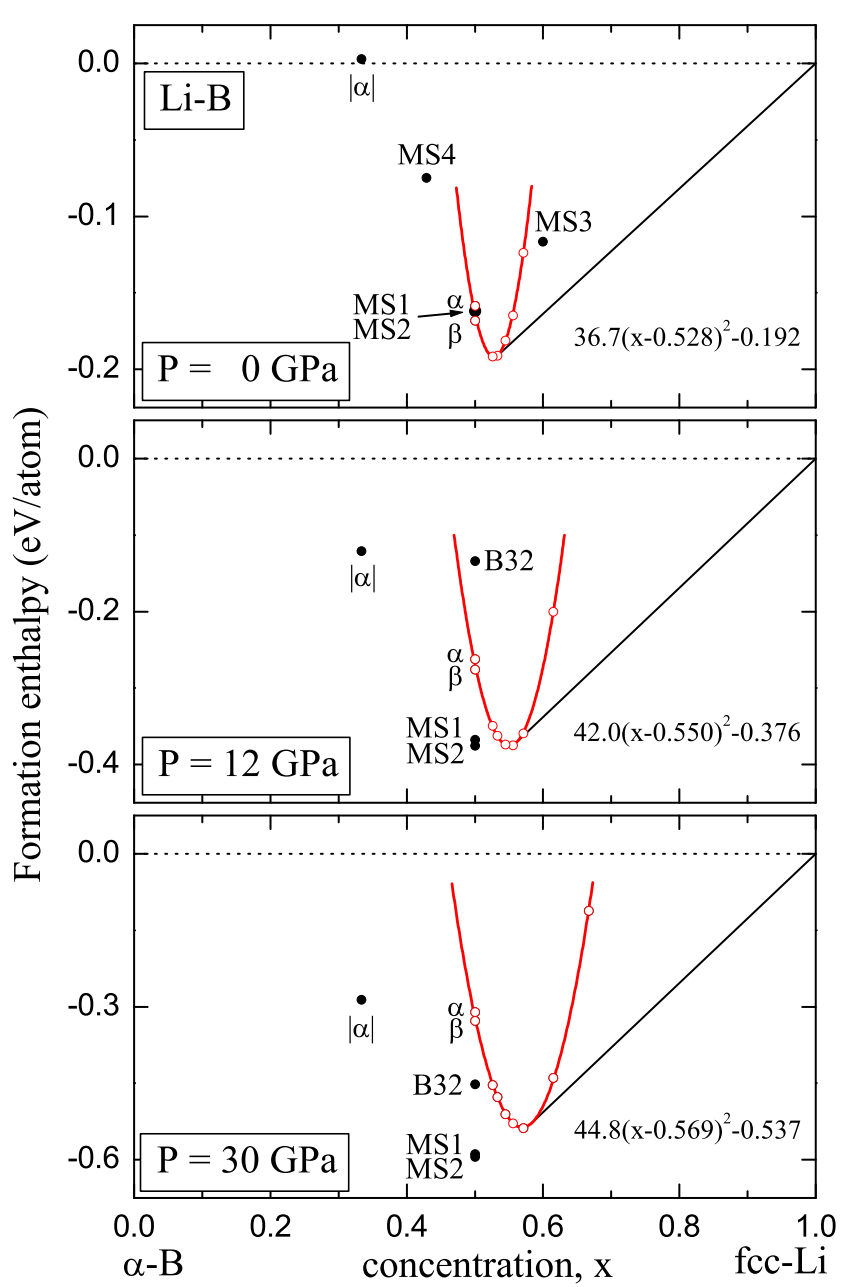

FIG. 6: (color online). Calculated phase diagrams for the Li-B system. Hollow red points are $\mathrm{M}_{2 n} \mathrm{~B}_{m}$ phases, from left to right: $\alpha$ - $\mathrm{LiB}, \beta-\mathrm{LiB}, \mathrm{Li}_{10} \mathrm{~B}_{9}, \mathrm{Li}_{8} \mathrm{~B}_{7}, \mathrm{Li}_{6} \mathrm{~B}_{5}, \mathrm{Li}_{10} \mathrm{~B}_{8}, \mathrm{Li}_{4} \mathrm{~B}_{3}$ for all pressures, $\mathrm{Li}_{8} \mathrm{~B}_{5}$ for $P=12 \mathrm{GPa}$, and $\mathrm{Li}_{8} \mathrm{~B}_{5}, \mathrm{Li}_{2} \mathrm{~B}$ for $P=30 \mathrm{GPa}$. The parameters of the parabolic fit are given on each panel; the solid lines are tangent to the parabolas at $x=0.534, x=0.560$, and $x=0.584$ for 0,12 , and 30 GPa pressure, respectively. $|\alpha|-\mathrm{LiB}$ is the $\mathrm{AlB}_{2}$ prototype and $\mathrm{B} 32$ is the pseudodiamond structure 38$]$. Note the different enthalpy scale for different pressures.

The allowed concentrations are in excellent agreement with the Wörle and Nesper's value of $y=0.9$ inferred from the analysis of the x-ray data 46$]$. Considering that the lithium and boron sublattices are nearly independent, it seems possible to manipulate the stoichiometry with active solutions by removing the alkali metal through the surface of the sample. By using tetrahydrofurannaphtalene solution Liu et al. may have extracted not only the free lithium, but also the lithium from the $\mathrm{LiB}_{y}$ compound pushing the concentration of boron towards the higher limit of the stability region $\left(y_{\min }, y_{\max }\right)$, and maybe beyond it.

To help determine the composition of the $\mathrm{LiB}_{y}$ com-

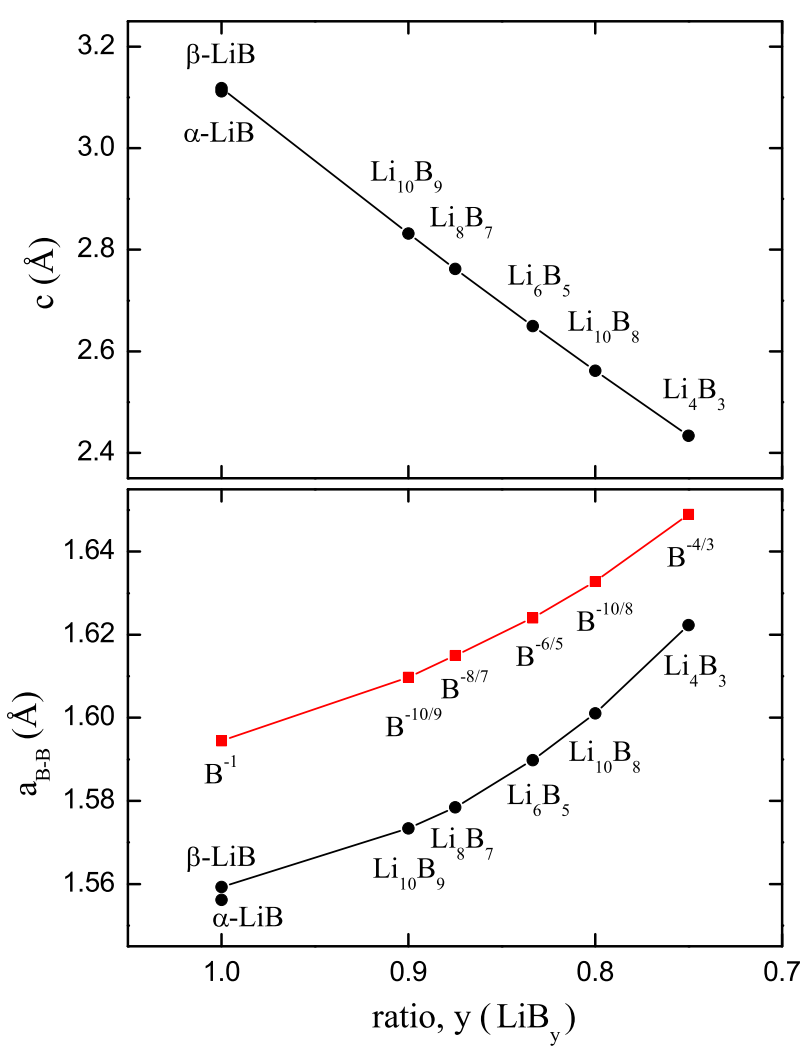

FIG. 7: (color online). Calculated optimized lattice parameters in phases with linear chains of boron. Top panel: $c$-axis distance (the doubled Li-Li interlayer spacing, $2 c_{L i-L i}$ ) as a function of the $\mathrm{B}$ to $\mathrm{Li}$ ratio in $\mathrm{LiB}_{y}$. Bottom panel: the black circles are the $\mathrm{B}-\mathrm{B}$ bond lengths in the $\mathrm{LiB}_{y}$ phases $\left(a_{B-B}=c / y / 2\right)$; the red squares are B-B bond lengths in the $\mathrm{B}^{-1 / y}$ phases explained in the text.

pounds from experimental data we plot the fully relaxed lattice parameters in Fig. 7. We observe that the $c$-axis undergoes an almost linear expansion with the increase of the boron to lithium ratio: $c=0.365+2.746 y$. Because of the $1 \sim 2 \%$ systematic errors in the bond length calculations within the GGA these results cannot be used to pinpoint the absolute value of $y$. However, the variation of the lattice parameters as a function of $y$ is expected to be much more accurate and allows one to estimate the range of concentrations for the experimentally observed compounds. For example, the measured $c=2.875(2)$ $\AA$ and $c=2.792(1) \AA$ values corresponded to nominal compositions $y=1.0$ and $y=0.82$, respectively 46 ; the slope from Fig. 7 indicates that the difference in $y$ in the synthesized compounds was, in fact, about 6 times smaller $(\Delta y=0.03)$. The discrepancy in the measured

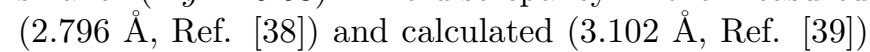
$c$-axis values pointed out by Rosner and Pickett [39] can be explained as that the synthesized compound was not a stoichiometric lithium monoboride but rather $\operatorname{LiB}_{y \approx 0.89}$. 


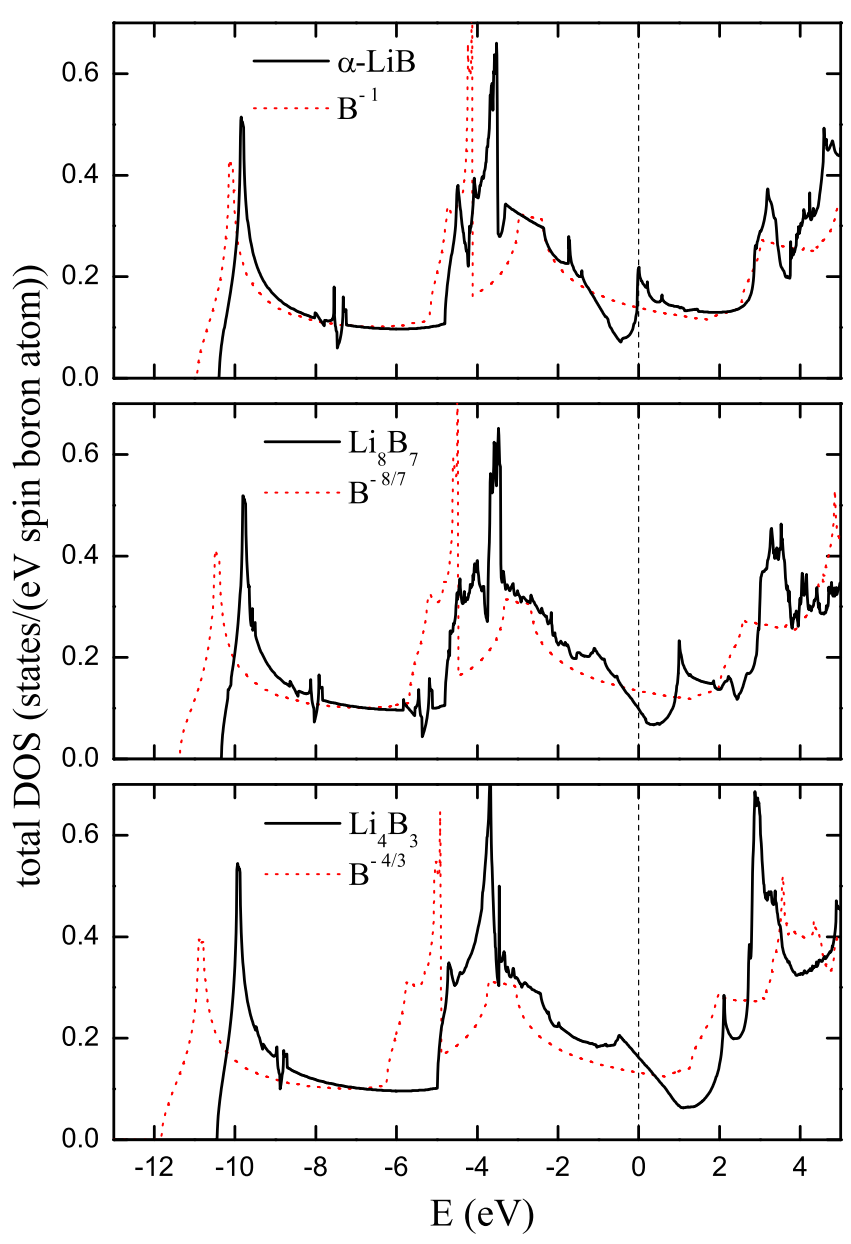

FIG. 8: (color online). Calculated total density of states (DOS) for the $\mathrm{Li}_{2 n} \mathrm{~B}_{m}$ compounds (solid black lines) and hypothetical $\mathrm{B}^{-1 / y}$ phases explained in the text (dotted red lines). Fermi level is at $0 \mathrm{eV}$.

It should be noted that the determination of the concentration $\left(y=a_{B-B} / c_{L i-L i}, c_{L i-L i}=c / 2\right)$ presents difficulty only because the length of the boron-boron bond is hard to extract from the experiment [46]. However, it is the covalent boron-boron bond, being very rigid, that determines the $c$-axis dimension. This can be best illustrated by simulating linear chains of boron in hypothetical structures $\mathrm{B}^{-1 / y}$ where lithium is replaced with the equivalent number of extra electrons $1 / y$ (a uniform positive background is used here to impose charge neutrality). The $\mathrm{B}^{-1 / y}$ structures with one boron atom per unit cell keep the basal lattice vectors of the corresponding $\mathrm{LiB}_{y}$ compounds while the $c$-axis is optimized. Fig. 7 shows that the charge transfer from lithium to boron alone can explain the variation of the bond length for $y=0.8 \sim 1.0$. The origin of the nearly constant offset between the two curves becomes evident when the lithium sublattice is, in turn, simulated without boron. A hypothetical $\mathrm{Li}^{+1}$ structure with the same in-plane di-

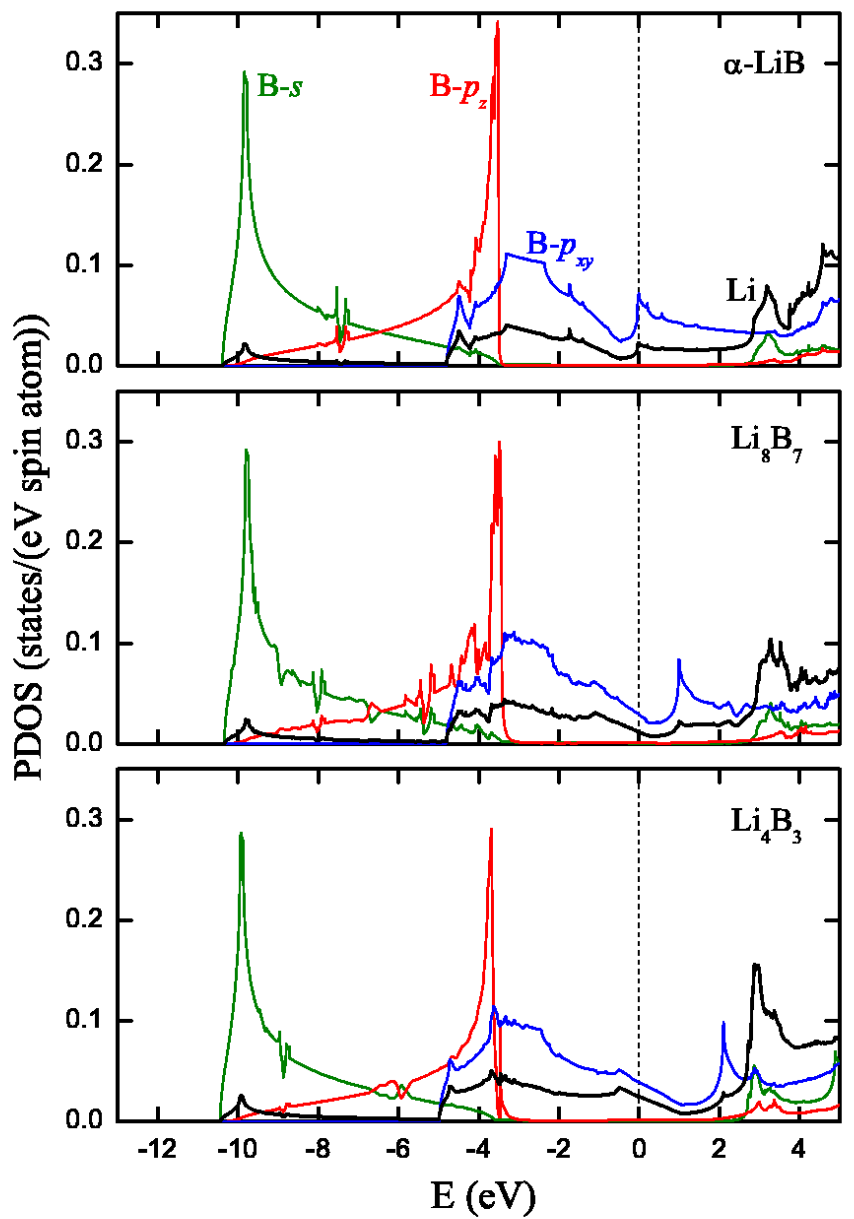

FIG. 9: (color online). Calculated partial density of states (PDOS) for the $\mathrm{Li}_{2 n} \mathrm{~B}_{m}$ compounds. The curves correspond to B- $s$ (green), B- $p_{z}$ (red), B- $p_{x y}$ (blue), and Li (black) characters. Fermi level is at $0 \mathrm{eV}$.

mensions as in $\alpha$-LiB has a much shorter equilibrium $c$ axis of $2.22 \AA$. The $E_{L i+1}(c)$ dependence in the $2.5 \sim 3.1 \AA$ range of $c$ is almost linear with a slope of $0.76 \mathrm{eV} / \AA$. As a result, the lithium sublattice in the $\mathrm{LiB}_{y}$ phases exerts a small stress on the linear chains of boron for all $y$ from 1 to 0.8 . The stress induces the shortening of the boronboron bond by $0.029 \AA(y=1)$ and $0.033 \AA(y=0.8)[49$ and thus turns out to be the main reason for the $0.035 \AA$ $(y=1)$ and $0.032 \AA(y=0.8)$ bond length mismatches in the simulated phases with and without lithium (Fig. 7). The result illustrates why $\mathrm{LiB}_{y}$ can be represented well as a superposition of the two electronically independent doped sublattices. However, our next test shows that lithium does play an important role in defining the optimal composition of $\mathrm{LiB}_{y}$ by affecting the electronic states of boron near the Fermi level.

To further investigate the mechanism leading to the existence of the off-stoichiometry lithium borides we plot the total and partial DOS for several $\mathrm{Li}_{2 n} \mathrm{~B}_{m}$ phases in 
Figs. 8 and 9 and the band structure in a representative $\alpha$-LiB phase in Fig. 10. The states near the Fermi level are hybridized $p \pi-\mathrm{B}$ and Li states 39 . The average presence of the Li character in the DOS in the -10 to $3 \mathrm{eV}$ energy range is small in $\alpha$ - $\operatorname{LiB}\left(N^{L i} / N_{p \pi}^{B} \approx 0.3\right)$, but becomes more significant in $\operatorname{Li}_{4} \mathrm{~B}_{3}\left(N^{L i} / N_{p \pi}^{B} \approx 0.5\right)$. The $p \pi$ boron states extend into the lithium-filled interstitials the furthest, so they are affected by the electrostatic potential from the lithium ions the most (in fact, the van Hove singularity in $\alpha$ - LiB at $E=0$ is not present in the corresponding $\mathrm{B}^{-1}$ structure described above, see dotted curves in Figs. 8 and 10). Note that the bonding and antibonding boron states are not well separated in the $\mathrm{LiB}_{y}$ phases: in the case of $\alpha$-LiB both types are present in the $0-1.3 \mathrm{eV}$ energy range (Fig. 10). Since the Fermi level in $\alpha$-LiB (Fig. 8) already catches the edge of the antibonding $p \pi$-B states, one would naturally expect for the compound to benefit from losing lithium. However, the system does not follow the rigid band scenario as the concentration of lithium increases: the van Hove singularity is pushed away from the Fermi level (in $\alpha$-LiB) to the right by over $2 \mathrm{eV}\left(\right.$ in $\mathrm{Li}_{4} \mathrm{~B}_{3}$ ) (see Fig. 8). The optimal position of the Fermi level near the bottom of the $p \pi$ pseudogap is achieved in $\mathrm{Li}_{10} \mathrm{~B}_{9}$ and $\mathrm{Li}_{8} \mathrm{~B}_{7}$, which is a part of the reason why the $\mathrm{LiB}_{y}$ phases have the minimum formation energy at $y \approx 0.9$. The correlation between the position of the Fermi level in the pseudogap and the maximum stability has been observed in various systems [4, 48].

Comparison of the MS phases with the known metal borides phases under pressure. To check whether there are more optimal charge transfer and strain conditions than those in MS1 and MS2 we simulate MS phases at other concentrations (Fig. 6, top panel). In the Li-B system the MS3 and MS4 phases have energies well above the $\alpha$-B $\leftrightarrow \alpha$-LiB and $\alpha$-LiB $\leftrightarrow$ fcc-Li tie-lines, so they will be unstable against phase separation into the known compounds (these MS phases remain metastable under hydrostatic pressure as well). This test confirms our earlier finding that MS1-LiB and MS2-LiB are particularly stable because of the near-optimal occupation of the binding boron states [9].

Our conjecture that the hydrostatic pressure would favor the MS lithium monoboride phases over the offstoichiometric $\mathrm{LiB}_{y}$ compounds $[9]$ is also supported by the results shown in Fig. 6. As far as the possibility of the MS-LiB formation is concerned, it is rather unfortunate that the $\mathrm{LiB}_{y}$ phases additionally stabilize by becoming more lithium rich under pressure. The information in Fig. 6 can be used to evaluate the minimum pressure required to stabilize MS2-LiB with respect to $\alpha$-B and $\mathrm{LiB}_{y}$. We estimate by interpolation that at $P_{\text {min }} \approx 5$ GPa the MS2-LiB phase lies on the line that goes through $x=0$ and is tangent to the $\mathrm{LiB}_{y}$ parabola. Therefore, MS2-LiB could appear in the experiment at $P>P_{\min }$ (not accounting for the possible systematic errors and

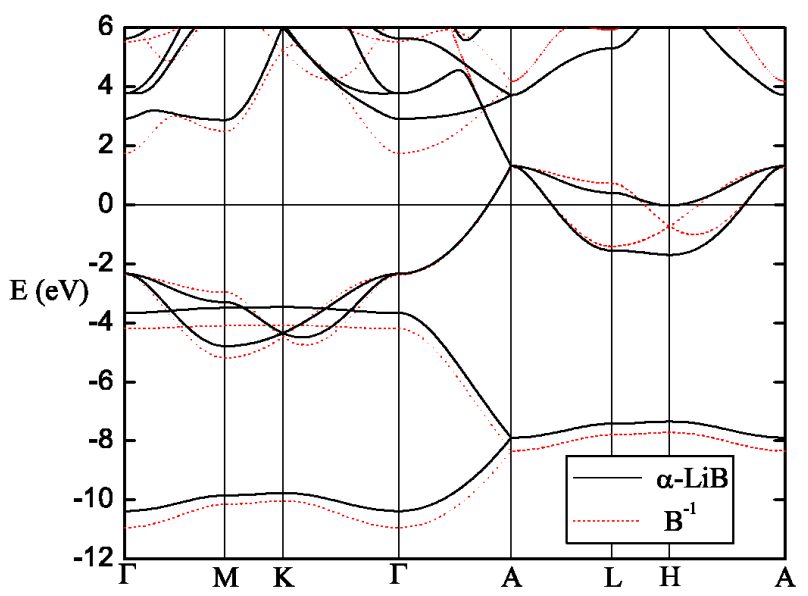

FIG. 10: (color online). Band structures of $\alpha-\mathrm{LiB}(38]$, solid black lines) and hypothetical $\mathrm{B}^{-1}$ (described in the text, dotted red lines) phases. To facilitate the comparison of the band structures in the two phases we doubled the $\mathrm{B}^{-1}$ unit cell along the $c$-axis and used the equilibrium lattice constants of $\alpha$-LiB. With the optimized $c$-axis the $\mathrm{B}^{-1}$ electronic states in the $-11 \sim-2 \mathrm{eV}$ range upshift by about $0.15 \mathrm{eV}$. Fermi level is at $0 \mathrm{eV}$.

the thermodynamics effects $\underline{9}$ ) if all other phases in the Li-B system were metastable under such pressures. Using the parabola coefficients for the three pressures given in Fig. 6 we also find by interpolation that MS2-LiB has the same formation enthalpy as the most stable $\mathrm{LiB}_{y \approx 0.82}$ phase at about 12 GPa. Finally, we observe that the line connecting MS2-LiB and fcc-Li crosses the parabolas in all the cases for pressures below $30 \mathrm{GPa}$ 50. This implies that if the MS2-LiB phase was synthesized, $\mathrm{LiB}_{y}$ might still be present in the sample as a by-product. For analysis of the Li-B system at higher pressures one needs to take into account that pure lithium undergoes phase transformations from fcc to $h R 1$ and eventually to $c I 16$ near $40 \mathrm{GPa} 51$.

The chances for the formation of the MS-LiB phases depend on where they are located on the phase diagram relative to $\mathrm{LiB}_{y}$ and the most lithium rich stable phase below $50 \%$ concentration. The known phases in this region have small atomic volume $\left(V_{L i_{3} B_{14}}=6.4\right.$ and $V_{L_{i B_{3}}}=7.5 \AA^{3} /$ atom under ambient conditions 13,36 , 37]) compared to MS2-LiB (11.2 and $6.7 \AA^{3} /$ atom at 0 and $30 \mathrm{GPa}$, respectively). The boron-rich phases could potentially bar the formation of MS-LiB under pressure, however they would need to have a very low formation enthalpy; for example, at $P=12 \mathrm{GPa}$ it would need to be below $H_{M S 2-L i B}=H_{L i B_{y} \approx 0.82}=-0.38 \mathrm{eV} /$ atom. Simulation of a known phase with $\mathrm{CaB}_{6}$ prototype, present in the K-B system 41, 53], could give information on how boron-rich phases respond to hydrostatic pressure. However, the ordered $\mathrm{CaB}_{6}-\mathrm{LiB}_{6}$ has a large atomic volume and actually becomes less stable under pressure: 

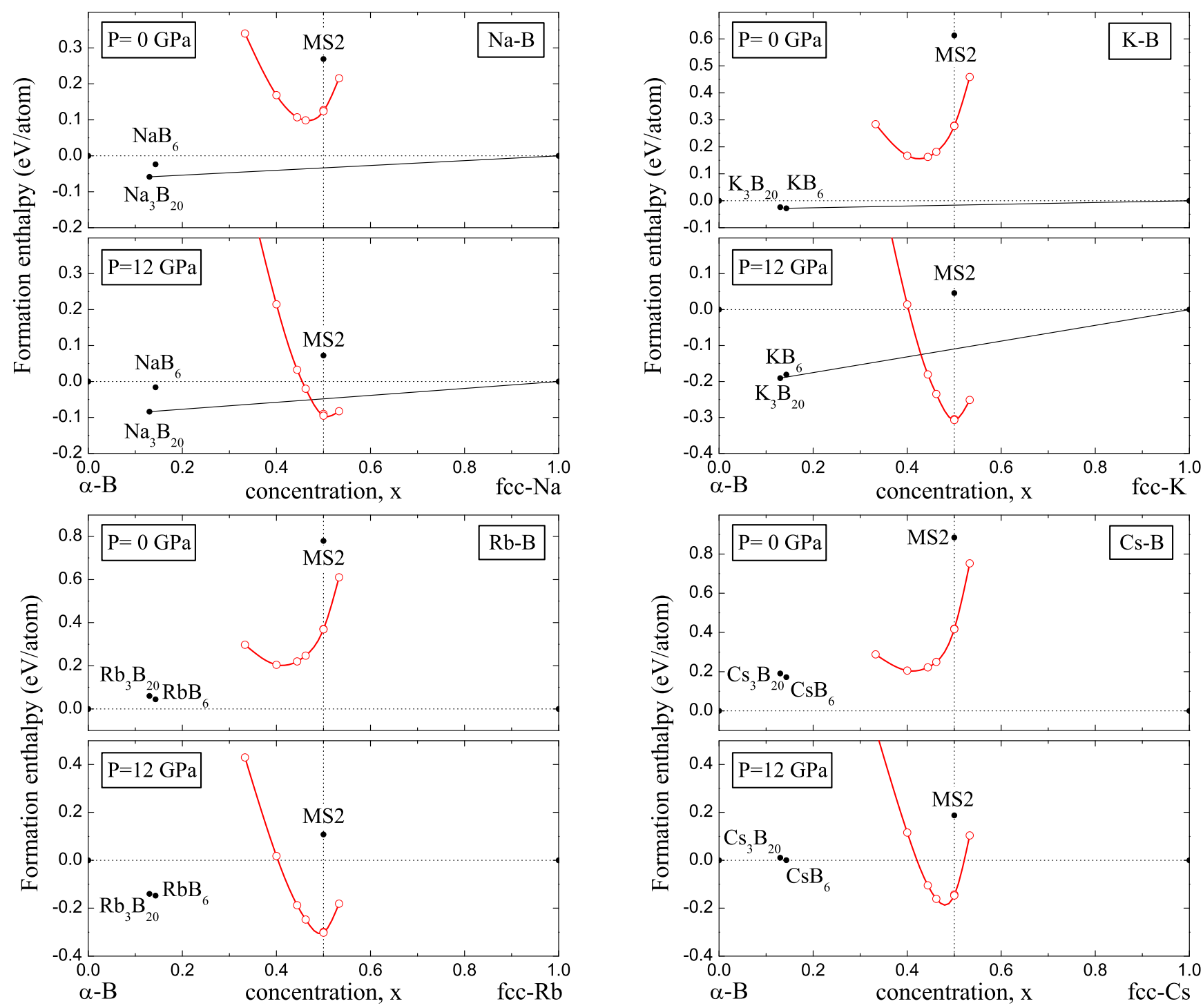

FIG. 11: (color online). Calculated phase diagrams for $\mathrm{Na}-\mathrm{B}$ and $\mathrm{Rb}-\mathrm{B}$ systems at different pressures. Hollow red points are $M_{2 n} B_{m}$ phases, from left to right: $M_{2} B_{4}, M_{2} B_{3}, M_{4} B_{5}$, $\mathrm{M}_{6} \mathrm{~B}_{7}, \alpha-\mathrm{MB}, \beta-\mathrm{MB}$, and $\mathrm{M}_{8} \mathrm{~B}_{7}$. The boron rich phases are $\mathrm{Na}_{3} \mathrm{~B}_{20}$ and $\mathrm{CaB}_{6}$ prototypes with Pearson symbols $o S 46$ and $c P 7$, respectively. The vertical dotted lines mark the $50 \%$ composition.

$H_{f}=0.016 \mathrm{eV} /$ atom, $V=10.1 \AA^{3} /$ atom at zero pressure and $H_{f}=0.303 \mathrm{eV} /$ atom, $V=8.6 \AA^{3} /$ atom at $30 \mathrm{GPa}$ pressure. Rosner and Pickett pointed out that a compact pseudodiamond phase B32 ( $\mathrm{NaTl}$ prototype) might appear under pressure 39. Using enthalpy versus pressure curves for the $\alpha-\mathrm{LiB}$ and $\mathrm{B} 32$ phases we find the crossover pressure to be $22 \mathrm{GPa}$. MS2-LiB stays below B32-LiB until about $65 \mathrm{GPa}$. Overall, our simulations suggest that there might be a window of pressures at which the MS-LiB phases can be synthesized.

FIG. 12: (color online). The same as in Fig. 11 for K-B and Cs-B systems.

\section{OTHER MONOVALENT AND SOME HIGHER-VALENT METAL BORIDES}

Alkali and transition metal borides. According to the experimental databases and the latest review of alkalimetal borides 13, 41] there are no stable sodium or potassium borides above $15 \%$ concentration of metal and no stable rubidium or cesium borides in the whole concentration range. $\mathrm{Na}_{3} \mathrm{~B}_{20}$ (Pearson symbol oS46) and $\mathrm{KB}_{6}$ (Pearson symbol $c P 7$ ) compounds, made out of boron polyhedra intercalated with alkali atoms, are considered the most metal-rich known borides in the Na-B and $\mathrm{K}$ B systems, respectively [13, 41, 52, 53]. Our simulations confirm that these compounds have negative formation enthalpies of $-58 \mathrm{meV} /$ atom for $\mathrm{Na}_{3} \mathrm{~B}_{20}$ and -29 
$\mathrm{meV} /$ atom for $\mathrm{KB}_{6}$ with respect to $\alpha$-B and fcc-M 35$]$. The formation Gibbs free energies for these compounds might be less negative if they were evaluated with respect to $\beta$-B at finite temperature 22]. This could be the reason why there is no conclusive evidence of potassium hexaboride synthesis 41]. Complete theoretical investigation of the boron-rich compounds such as $\mathrm{Na}_{3} \mathrm{~B}_{29}$ (Ref. [54]) is beyond the scope of this study but it is interesting to see where the nearly stoichiometric $\mathrm{MB}_{y}$ phases place with respect to the known phases in these systems.

As in the $\mathrm{LiB}_{y}$ compounds, the metal and boron sublattices in the alkali boride phases $\mathrm{MB}_{y}(\mathrm{M}=\mathrm{Na}, \mathrm{K}, \mathrm{Rb}$, $\mathrm{Cs}$ ) are found to be very weakly correlated. In fact, the larger alkali atoms push the boron chains farther apart (see Table I) weakening the boron interchain bonds (note the lower energy difference between $\alpha$-MB and $\beta$-MB listed in Table I). This again leads to the situation when $\mathrm{MB}_{y}(\mathrm{M}=\mathrm{Na}, \mathrm{K}, \mathrm{Rb}, \mathrm{Cs})$ compounds can easily adapt to an optimal composition by having incommensurate metal and boron sublattices. Figures 11 and 12 demonstrate that in all Na-B, K-B, Rb-B, Cs-B systems the stoichiometric phases with linear chains of boron prefer to lose some metal, the opposite tendency compared to the Li-B system. While the most stable $\mathrm{LiB}_{y}$ composition appears to be determined primarily by the optimal level of boron doping, in the larger alkali-metal borides, $\mathrm{MB}_{y}$, the lattice mismatch between the metal and boron sublattices must be playing a more significant role. Note that the formation enthalpy points for these $\mathrm{M}_{2 n} \mathrm{~B}_{m}$ phases are not symmetric, curving up more rapidly in the metal rich region.

The stabilization from losing a few percent of alkali metal is noticeable but not enough for $\mathrm{NaB}_{y}$ and $\mathrm{KB}_{y}$ to have a negative formation enthalpy at zero pressure. Because the interchain spacing is determined mostly by the alkali cations the $C_{11}+C_{12}$ force constant in $\beta$-MB decreases as one moves down the periodic table (see Table I). $C_{33}$ also becomes smaller as the boron-boron bond length gets longer. The softness of the $\mathrm{MB}_{y}$ phases $(\mathrm{M}=$ $\mathrm{Na}, \mathrm{K}, \mathrm{Rb}, \mathrm{Cs}$ ) invites the use of hydrostatic pressure for their synthesis. Moreover, in the Na-B and K-B systems the $\mathrm{MB}_{y}$ phases stabilize more rapidly than the MS2-MB phases, which makes it unlikely for the latter to form under the pressures considered. Synthesis of $\mathrm{MB}_{y}(\mathrm{M}=$ $\mathrm{Na}, \mathrm{K}, \mathrm{Rb}, \mathrm{Cs}$ ) would provide valuable information on the ways the linear chains of boron could be stabilized. Because the alkali-metal borides are not fully explored, it would not be surprising if a not considered here or a completely unknown phase appeared in such an experiment.

While the $\beta$-MB phases are only metastable for the borides in the alkali-metal series, there are two reported stable transition-metal monoborides in this configuration: RhB and PtB (Ref. [13, 55]). Our fully relaxed unit cell parameters (see Table I) agree well with experiment for $\beta$-RhB $(a=3.309 \AA, c=4.224 \AA)$, but they dis- agree by over $10 \%$ with the measured values $(a=3.358$ $\AA, c=4.058 \AA$ ) for $\beta$-PtB [13, 55]. Identification of the source of this discrepancy requires additional study of this system. Nevertheless, the data on $\beta-\mathrm{RhB}$ and $\beta$ $\mathrm{PtB}$ in Table I give an idea about what difference the $d$ electrons cause in the boron-boron binding compared to the case of the alkali-metal monoborides. For example, $\beta$-RhB and $\beta$-PtB no longer have the optimally doped double-bonded boron chains: the boron-boron bond is so overstretched that it exceeds the $s p^{2}$ bond length in the $\mathrm{AlB}_{2}$-type compounds, resulting in the increase of the $c$-axis compressibility compared to the alkali-metal monoborides. The significant reduction of the interchain distances leads to an over $300 \%$ increase in the $C_{11}+C_{12}$ force constants. One more important consequence of the more compact arrangement of atoms in the lateral direction and the hybridization of the $d$-orbitals of metal with the valence states of boron is the much larger energy difference between the $\beta$-MB and $\alpha$-MB structures. This makes the formation of the off-stoichiometry phases with linear chains of boron in the transition-metal monoborides energetically unfavorable.

Noble- and divalent-metal borides. $\mathrm{AgB}_{2}$ and $\mathrm{AuB}_{2}$ have been shown to have big positive formation energies [4], so they should not form at ambient pressure. Recent experiments suggest that some superconducting Ag-B phase was formed by pulsed laser deposition [12]. Because of the synthesis conditions, the thin-film samples were inhomogeneous and did not produce new x-ray peaks. It was assumed that the observed phase was $|\alpha|$ prototype, although the $T_{c}$ turned out to be much lower than the anticipated value[1]. Formation of $\mathrm{Ag}$ vacancies in $|\alpha|-\mathrm{Ag}_{x} \mathrm{~B}_{2}$ was suggested by Shein et al. as a possible explanation of the observed data 10]. Current simulations offer other possibilities for formation of phases under non-equilibrium conditions: the proposed phases still have positive formation energies but they are less unstable and below the respective $|\alpha| \leftrightarrow$ metal tie-lines (Fig. 13). The intermediate phases in the concentration range from $33 \%$ to $60 \%$ generally stay below the tie-lines.

TABLE I: Calculated properties of $\beta$-MB metal borides (NiAs prototype).

\begin{tabular}{c|ccccc}
\hline \hline compound & $\begin{array}{c}a_{0} \\
(\AA)\end{array}$ & $\begin{array}{c}c_{0} \\
(\AA)\end{array}$ & $\begin{array}{c}C_{11}+C_{12} \\
(\mathrm{GPa})\end{array}$ & $\begin{array}{c}C_{33} \\
(\mathrm{GPa})\end{array}$ & $\begin{array}{c}E_{\beta-M B}-E_{\alpha-M B} \\
(\mathrm{meV} / \text { atom })\end{array}$ \\
\hline $\mathrm{LiB}$ & 4.013 & 3.120 & 139 & 542 & -10 \\
$\mathrm{NaB}$ & 4.697 & 3.196 & 111 & 379 & -3.1 \\
$\mathrm{~KB}$ & 5.390 & 3.240 & 79 & 268 & -1.6 \\
$\mathrm{RbB}$ & 5.662 & 3.267 & 77 & 251 & -1.7 \\
$\mathrm{CsB}$ & 5.976 & 3.325 & 69 & 252 & -2.0 \\
\hline $\mathrm{RhB}$ & 3.382 & 4.185 & 498 & 309 & -267 \\
$\mathrm{PtB}$ & 3.765 & 3.655 & 546 & 293 & -108 \\
\hline \hline
\end{tabular}




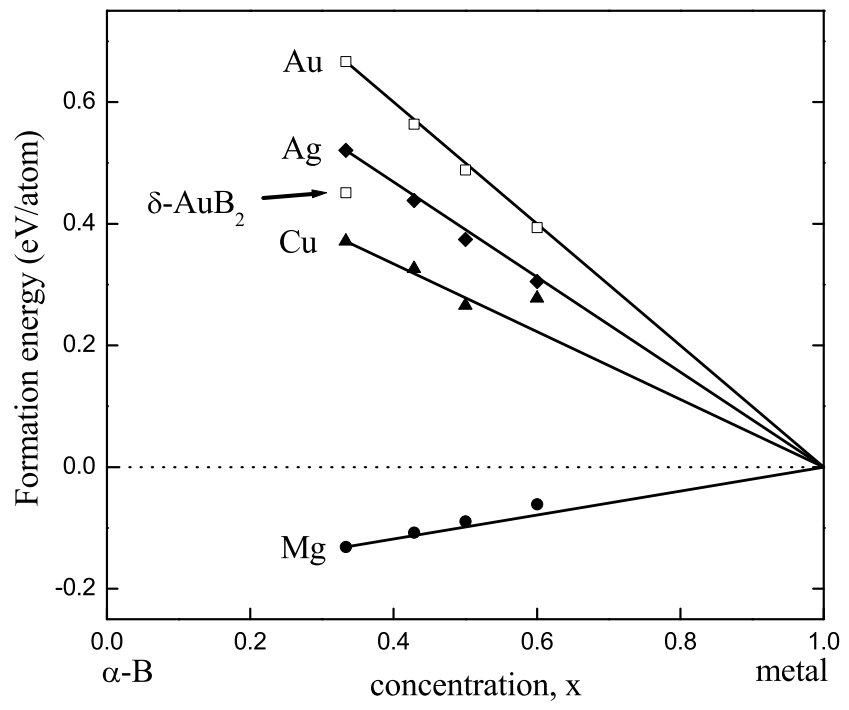

FIG. 13: (color online). Calculated phase diagrams for magnesium and noble-metal borides. The solid points are layered phases from left to right: $|\alpha|$, MS4, MS2, MS3. We also show $\delta$-AuB $B_{2}$ phase [32], which is significantly lower than $|\alpha|-\mathrm{AuB}_{2}$.

If formed, MS2-AgB would be even more dynamically stable than MS2-LiB: we find that the former represents a stable equilibrium with the frequencies of the softest interlayer modes $\omega_{x, y}$ three times higher than those in the latter $[9]$. This is an expected result, considering that the silver bilayer in MS2-AgB remains bound by the $d$ electrons even if it donates most of the charge from the $s$-orbital to boron as lithium does. The electronic properties and the stability of the MS noble-metal borides are further discussed in Section 7.

We find that the $\delta-\mathrm{MB}_{2}$ phase 32 is surprisingly much more stable than the $|\alpha|-\mathrm{MB}_{2}$ phase for several metals: $\mathrm{Au}, \mathrm{Ca}, \mathrm{K}, \mathrm{Pd}$, and Pt (by 215, 28, 214, 28, and 272 $\mathrm{meV} /$ atom, respectively). Apparently, metal atoms prefer to hybridize their valence states with $p$-orbitals of boron more strongly by shifting to a boron-boron bond, rather than simply donate their valence electrons. Note that the five metals are either large in size $\left(d_{C a}=3.88\right.$ $\AA, d_{K}=4.71 \AA$, see Fig. 4) or have a big work function (bulk values: $\phi_{A u}=5.1 \mathrm{eV}, \phi_{P d}=5.12 \mathrm{eV}, \phi_{P t}=5.65$ $\mathrm{eV}[56,[57])$. The discovery of the lower-symmetry $\delta$ $\mathrm{AuB}_{2}$ phase 32$]$ rules out the possibility of $|\alpha|-\mathrm{AuB}_{2}$ synthesis. The formation energy of $\delta-\mathrm{AuB}_{2}$ remains positive $(0.45 \mathrm{eV} /$ atom, see Fig. 13), which makes this phase unlikely to form as well. In the Ca-B system $\delta-\mathrm{CaB}_{2}$ is still unstable against phase separation into $\mathrm{CaB}_{6}$ and fcc-Ca [4, 13] by $144 \mathrm{meV} /$ atom.

The MS phases in the Mg-B system stay at least a few $\mathrm{meV} /$ atom above the $|\alpha|-\mathrm{MgB}_{2} \leftrightarrow \mathrm{hcp}-\mathrm{Mg}$ tie-line. Hydrostatic pressure is insignificant to their relative stability because they compete against similar phases. Therefore, the MS magnesium boride phases are not likely to form and could possibly exist only in the form of a defect in $|\alpha|-\mathrm{MgB}_{2}$. We calculate the following series: $|\alpha \beta|$, $|\alpha| \alpha \beta|,| \alpha|\alpha| \alpha \beta \mid$, and so on up to $8|\alpha|$ unit cells, and find by extrapolation that the energy required to insert a single magnesium layer into the $|\alpha|-\mathrm{MgB}_{2}$ matrix is quite high: over $25 \mathrm{meV}$ per atom in the additional layer of magnesium.

\section{SUMMARY}

Electronic properties. It is illustrative to compare the important features of the electronic structure in the lowvalent metal borides to those in the lithium borides, which were discussed in our previous study $[9]$. We focus mainly on the $p$-states of boron, important for the stability and the superconductivity in these compounds. For convenience, we calculate the band structure and PDOS for $|\alpha|$, MS2, and MS3 phases because all three have a hexagonal unit cell. The key characteristics of the boron states, along with parameters of the unit cells for these

TABLE II: Metal boride phases: formation energy $\left(E_{f}\right.$, $\mathrm{eV} /$ atom $)$, relative stability $\left(\Delta E_{X}, \mathrm{eV} /\right.$ atom, Eq. 1), inplane boron-boron bond $\left(a_{B-B}, \AA\right)$, interplanar boron-boron distance $\left(c_{B-B}, \AA\right)$, internal coordinate of the metal atom $\left(z_{M}\right)$ [28, [29], position of $p \sigma$-band in boron at $\Gamma$ and $A \mathrm{k}$ points $\left(E_{\Gamma}, E_{A}, \mathrm{eV}\right)$, and PDOS at $E_{F}$ for B- $p_{x y}\left(N_{p_{x y}}^{B}(0)\right.$, states $/(\mathrm{eV} \cdot \mathrm{spin} \cdot$ boron atom $))$.

\begin{tabular}{|c|c|c|c|c|c|c|}
\hline phase & & $\mathrm{Li}$ & $\mathrm{Ag}$ & $\mathrm{Au}$ & $\mathrm{Cu}$ & $\mathrm{Mg}$ \\
\hline \multirow{6}{*}{$\begin{array}{c}|\alpha| \\
(33 \%)\end{array}$} & $E_{f}$ & 0.003 & 0.520 & 0.667 & 0.371 & -0.131 \\
\hline & $a_{B-B}$ & 1.717 & 1.744 & 1.737 & 1.721 & 1.776 \\
\hline & $c_{B-B}$ & 3.469 & 4.081 & 4.260 & 3.382 & 3.521 \\
\hline & $E_{\Gamma}$ & 1.48 & 1.29 & 1.26 & 1.14 & 0.39 \\
\hline & $E_{A}$ & 1.70 & 1.01 & 0.95 & 0.65 & 0.77 \\
\hline & $N_{p_{x y}}^{B}(0)$ & 0.076 & 0.090 & 0.093 & 0.106 & 0.049 \\
\hline \multirow{8}{*}{$\begin{array}{c}\text { MS2 } \\
(50 \%) \\
{[28]}\end{array}$} & $E_{f}$ & -0.162 & 0.374 & 0.488 & 0.266 & -0.089 \\
\hline & $\Delta E_{M S 2}$ & -0.164 & -0.016 & -0.012 & -0.013 & 0.009 \\
\hline & $a_{B-B}$ & 1.765 & 1.739 & 1.734 & 1.734 & 1.805 \\
\hline & $c_{B-B}$ & 5.522 & 6.369 & 6.589 & 4.915 & 5.989 \\
\hline & $z_{M}$ & 0.496 & 0.368 & 0.356 & 0.388 & 0.440 \\
\hline & $E_{\Gamma}$ & 0.99 & 1.19 & 1.17 & 0.69 & 0.15 \\
\hline & $E_{A}$ & 0.99 & 1.19 & 1.17 & 0.69 & 0.15 \\
\hline & $N_{p_{x y}}^{B}(0)$ & 0.059 & 0.086 & 0.091 & 0.091 & 0.043 \\
\hline \multirow{8}{*}{$\begin{array}{c}\text { MS3 } \\
(60 \%) \\
{[29]}\end{array}$} & $E_{f}$ & -0.117 & 0.305 & 0.394 & 0.277 & -0.061 \\
\hline & $\Delta E_{M S 3}$ & -0.118 & -0.007 & -0.006 & 0.054 & 0.018 \\
\hline & $a_{B-B}$ & 1.745 & 1.731 & 1.722 & 1.695 & 1.816 \\
\hline & $c_{B-B}$ & 8.318 & 8.785 & 9.097 & 6.903 & 8.511 \\
\hline & $z_{M}$ & 0.178 & 0.231 & 0.237 & 0.228 & 0.194 \\
\hline & $E_{\Gamma}$ & 1.23 & 1.22 & 1.25 & 0.88 & 0.03 \\
\hline & $E_{A}$ & 1.23 & 1.22 & 1.25 & 0.89 & 0.03 \\
\hline & $N_{p_{x y}}^{B}(0)$ & 0.066 & 0.090 & 0.098 & 0.074 & 0.039 \\
\hline
\end{tabular}


phases are given in Table II.

For all MS2 metal borides the $p \sigma$ band along $\Gamma$-A is practically flat because of the large separation between boron layers, as shown in Fig. 4 of Ref. [9] for MS2-LiB and in Fig. 14 for MS2-AgB. For Ag and Au this band does not move much from the respective average positions in $|\alpha|$ and the PDOS of $p \sigma$ states in boron at the Fermi level, $N_{p_{x y}}^{B}(0)$, stays nearly the same (Table II and Fig. 15). In fact, these boron properties remain the same in the more metal-rich structure MS3. The results suggest that the level of doping of the boron layers in the MS noble metal borides is nearly independent of the number of metal layers. Considering this and the fact that the MS phases in the 33\%-100\% range closely follow the $|\alpha| \leftrightarrow$ fcc lines on the Ag-B and Au-B phase diagrams these MS phases can be viewed as a mixture of weakly interacting building blocks: the $|\alpha|$ unit cell with an established charge redistribution within it and the closedpacked layers of pure metal. Therefore, one could expect the superconducting properties of the boron layer in the hypothetical phases $|\alpha|-\mathrm{AgB}_{2}$ and the MS silver borides in the $33 \%-100 \%$ concentration range to be similar. However, the nonequilibrium conditions necessary to synthesize such compounds 12 may introduce disorder destroying the superconducting states [8].

Compared to the MS gold and silver borides, the MS copper borides deviate more from the $|\alpha|-\mathrm{CuB}_{2} \leftrightarrow$ fcc- $\mathrm{Cu}$ line (Fig. 13) and the level of boron doping is more nonmonotonic as a function of the metal concentration (Table II), even though the work functions of $\mathrm{Cu}$ and $\mathrm{Ag}$ are close (bulk values: $\phi_{C u}=4.65 \mathrm{eV}, \phi_{A g}=4.26 \mathrm{eV}$, Ref. [56]). The different behavior must be the result of the more pronounced strain between the copper and boron layers caused by the smaller size of copper $\left(d_{C u}=2.563\right.$ $\AA, d_{A g}=2.937 \AA, d_{A u}=2.949 \AA$, see Fig. 4). In the more electron-rich Mg-B system the level of boron doping increases as additional magnesium layers are added. In MS3- $\mathrm{Mg}_{3} \mathrm{~B}_{2}$ the $p \sigma$ states are almost completely occupied.

Lithium turns out to be a special case among the monovalent metals: it has the right size and can easily donate electrons to stabilize the boron layers in the MS phases. Fig. 6 and Table II show that the stabilization effect is largest for the $50 \%$ concentration MS1 and MS2 lithium borides. No less importantly, by giving up most of their charge the lithium layers interact only weakly with one another, which makes the compound very soft along the $c$-axis and gives the opportunity to stabilize the compound even more with hydrostatic pressure [9]. The addition of extra layers does not result in further stabilization, as boron in MS3- $\mathrm{Li}_{3} \mathrm{~B}_{2}$ is doped less than in MS2-LiB, judging by the location of the $p \sigma$ band along $\Gamma$-A (Table II and Fig. 15). The present PAW calculations confirm our previous result that MS2-LiB has a higher PDOS of boron $p \sigma$ states at the Fermi level than that in $\mathrm{MgB}_{2}$ (Ref. [9]). Note that the PDOS in our sim-

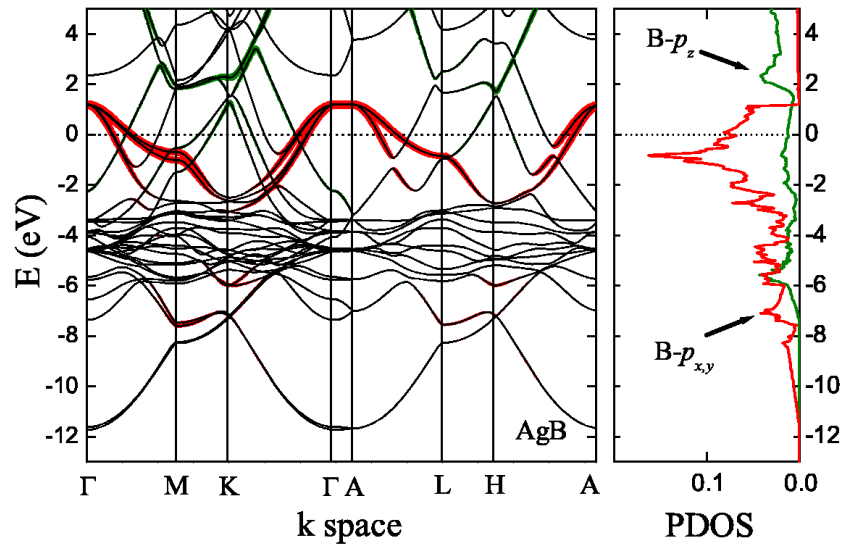

FIG. 14: (color online). Band structure and partial density of states (PDOS) in a hypothetical MS2-AgB phase, calculated in APW+lo 21, 58]. PDOS units are states/(eV ·spin) per boron atom. The thickness of band structure lines is proportional to boron $p_{x, y}$ (red) and $p_{z}$ (green) characters. Fermi level is at $0 \mathrm{eV}$.

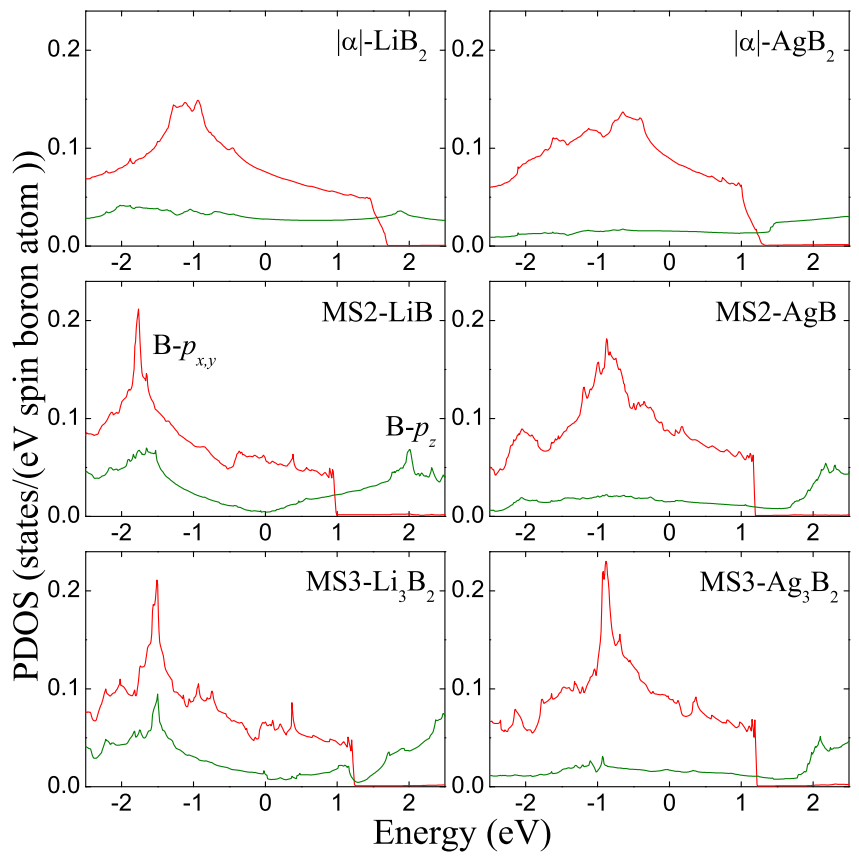

FIG. 15: (color online). PDOS of boron $p_{x, y}$ (red) and $p_{z}$ (green) states in lithium and silver MS borides calculated with VASP. Fermi level is at $0 \mathrm{eV}$.

ulations is found by decomposition of the wavefunction within a sphere of fixed radius and can slightly vary with this parameter, as well as with the approximation used. In the APW+lo calculation we obtained a $12 \%$ increase of $N_{p_{x y}}^{B}(0)$ in MS2-LiB compared to that in $\mathrm{MgB}_{2}$ using $R_{M T}^{B}=1.6$ a.u. [9], while in the present PAW calculations we observe a $20 \%$ increase using the default PAW radius of 1.7 a.u. 
The ample amount of the boron $p \sigma$ states at the Fermi level in MS2-LiB holds great promise for this compound to be a good superconductor. However, a more thorough calculation of the electron-phonon coupling, such as in Ref. [60], is required to say with certainty whether the new lithium monoboride can compete with the recordholder $\mathrm{MgB}_{2}$. Such calculation is underway 61 .

$X$-ray reflections. As we pointed out previously $[9]$, the resulting structure in metal borides at 1:1 composition could be a random mixture of MS1 and MS2, because they differ only by a long-period shift in stacking order and, therefore, are nearly degenerate. The two phases also have very close nearest neighbor distances in most systems and random structures would still have a constant separation between boron layers. For magnesium and silver MS monoborides these periods correspond to $2 \theta=16.4^{\circ}$ and $2 \theta=16.8^{\circ}$, respectively (for $\lambda=1.5418$ $\AA)$.

The largest difference (3\%) in the c-axis for MS1 and MS2 is actually found for Li-B, resulting in $2 \theta=16.6^{\circ}$ $\left(\mathrm{d}_{M S 1-L i B}=5.35 \AA\right)$ and $2 \theta=16.1^{\circ}\left(\mathrm{d}_{M S 2-L i B}=5.52\right.$ $\AA)$; the more lithium-rich phase $\mathrm{MS} 3-\mathrm{Li}_{3} \mathrm{~B}_{2}$ would produce a reflection at $2 \theta=10.6^{\circ}\left(\mathrm{d}_{M S 3-L i_{3} B_{2}}=8.32\right.$ $\AA$ ). The most pronounced peaks in the published x-ray data for lithium monoboride are at $2 \theta=25.5^{\circ}, 41.3^{\circ}$, and $45.0^{\circ}$ which fit well to the calculated $\alpha$ - $\mathrm{LiB}$ x-ray pattern [38]. Interestingly, two reflections at low angles $2 \theta=12.2^{\circ}$ and $20.9^{\circ}$ were observed at $40-50 \%$ of lithium concentration 42. A low-angle reflection was also detected at $12.8^{\circ}$ in $\mathrm{Al}_{1-x} \mathrm{Li}_{x} \mathrm{~B}_{2}$ under heavy $\mathrm{Li}$ doping $[59$. However, none of the observed peaks in the samples prepared at ambient pressure match the calculated x-ray reflections in the MS lithium borides.

\section{CONCLUSIONS}

The main results of the present study can be summarized as follows:

i. We have identified a previously unknown class of metal-rich layered phases that are comparable in energy to existing metal borides. This interesting accidental result should be credited to the exhaustive consideration of all candidates in the DMQC method and the careful structural relaxation in the calculation of their ground state energies.

ii. Our $a b$ initio results suggest that the MS phases are most suitable for electron-deficient metal boride systems. In the Ag-B and Au-B systems the MS phases are less unstable than the corresponding diborides with the $\mathrm{AlB}_{2}$ prototype but they still have positive formation energies. In the Mg-B system the MS phases are metastable and could possibly exist only as a defect in $\mathrm{MgB}_{2}$.

iii. The MS-LiB phases present a special case among the MS metal borides: lithium has the right size and valence to stabilize the hexagonal layers of boron at 1:1 composition. The MS lithium monoboride phases are shown to have lower formation enthalpy with respect to the experimentally observed nearly stoichiometric $\mathrm{LiB}_{y}$ phases under hydrostatic pressure. This encouraging result suggests that the new superconducting MS-LiB phases might form under proper conditions. The lowest required pressure depends on the position of boron-rich phases in the Li-B phase diagram and could be as low as several GPa.

iv. For a more complete description of the Li-B system we introduce a simple model of the off-stoichiometric $\mathrm{LiB}_{y}$ phases which explains the available experimental data. We demonstrate that because of the weak correlation between the boron and lithium sublattices the compound can easily adapt to an optimal composition, which corresponds to an optimal level of boron doping with the Fermi level lying near the bottom of the pseudogap. Interestingly, these phases turn out to be stable over a range of concentrations around $y=0.9$, in excellent agreement with experiment. We list the relaxed unit cell parameters which should be helpful in the determination of the $\mathrm{LiB}_{y}$ composition.

v. We consider the $\mathrm{MB}_{y}$ phases for other alkali-metal borides and find that these compounds also benefit from going off-stoichiometry, only in this case they prefer to lose some metal. The ensuing gain in enthalpy does not make them stable under ambient conditions, however these phases might form under hydrostatic pressure. Synthesis of the $\mathrm{MB}_{y}$ phases $(\mathrm{M}=\mathrm{Na}, \mathrm{K}, \mathrm{Rb}, \mathrm{Cs})$ would provide valuable information on how linear chains of boron could be stabilized.

We thank M. Calandra, F.H. Cocks, V. Crespi, P. Lammert, E. Margine, and J. Sofo for valuable discussions. We acknowledge the San Diego Supercomputer Center for computational resources.

[1] J. Nagamatsu, N. Nakagawa, T. Muranaka, Y. Zenitani, and J. Akimitsu, Nature 410, 63 (2001).

[2] J. Kortus, I.I. Mazin, K.D. Belashchenko, V.P. Antropov, and L.L. Boyer, Phys. Rev. Lett. 86, 4656 (2001).

[3] I.R. Shein, N.I. Medvedeva, and A.L. Ivanovskii, Phys. Solid State, 432213 (2001).

[4] T. Oguchi, J. Phys. Soc. Jpn. 71, 1495 (2002).

[5] R.J. Cava, H.W. Zandbergen, and K. Inumaru, Physica C 385, 8 (2003).

[6] P. Zhang, S.G. Louie, and M.L. Cohen, Phys. Rev. Lett. 94, 225502 (2005).

[7] H. Rosner, A. Kitaigorodsky, and W.E. Pickett, Phys. Rev. Lett. 88, 127001 (2002).

[8] A.M. Fogg, J.B. Claridge, G.R. Darling and M.J. Rosseinsky, Chemical Communications 121348 (2003).

[9] A.N. Kolmogorov and S. Curtarolo, Phys. Rev. B 73, 180501(R) (2006).

[10] I.R. Shein, N.I.Medvedeva, and A.L. Ivanovskii, cond-mat/0412426 (2004). 
[11] S.K. Kwon, S.J. Youn, K.S. Kim, and B.I. Min, cond-mat/0106483 (2001).

[12] R. Tomita, H. Koga, T. Uchiyama, and L. Iguchi, J. Phys. Soc. Jpn. 73, 2639 (2004).

[13] P. Villars, K. Cenzual, J. L. C. Daams, F. Hulliger, T. B. Massalski, H. Okamoto, K. Osaki, A. Prince, and S. Iwata, Crystal Impact, Pauling File. Inorganic Materials Database and Design System, Binaries Edition, ASM International, Metal Park, OH (2003)

[14] To facilitate comparison with experiment we use the $\mathrm{LiB}_{y}$ notation for boron-deficient lithium monoboride phases. Concentration of boron is denoted as $y$ instead of $x$ to avoid confusion with the alternative notation $\mathrm{Li}_{x} \mathrm{~B}_{1-x}$ $(y=(1-x) / x)$.

[15] G. Kresse and J. Hafner, Phys. Rev. B 47, 558 (1993).

[16] G. Kresse and J. Furthmuller, Phys. Rev. B 54, 11169 (1996).

[17] P.E. Blochl, Phys. Rev. B 50, 17953 (1994).

[18] J.P. Perdew, K. Burke, and M. Ernzerhof, Phys. Rev. Lett. 773865 (1996).

[19] G. Kresse and D. Joubert, Phys. Rev. B 59, 1758 (1999).

[20] J.D. Pack and H.J. Monkhorst, Phys. Rev. B 13, 5188 (1976); 16, 1748 (1977).

[21] An augmented plane wave+local orbitals program for calculating crystal properties P. Blaha, K. Schwarz, G.K.H. Madsen, D. Kvasnicka, and J. Luitz, WIEN2K, Karlheinz Schwarz, Technical Universitdt Wien, Austria, 2001.

[22] A. Masago, K. Shirai, and H. Katayama-Yoshida, Phys. Rev. B 73, 104102 (2006).

[23] S. Curtarolo, D. Morgan, K. Persson, J. Rodgers, and G. Ceder, Phys. Rev. Lett. 91, 135503 (2003).

[24] D. Morgan, G. Ceder, and S. Curtarolo, Meas. Sci. Technol. 16, 296 (2005).

[25] Description of the methods and calculation details can be found in [23].

[26] R. Magri, S.-H. Wei, and A. Zunger, Phys. Rev. B 42, 11388 (1990). V. Ozolins, C. Wolverton, and A. Zunger, Phys. Rev. B 57, 6427 (1998).

[27] MS1: 4 atoms in the primitive unit cell, space group $\mathrm{R} \overline{3} \mathrm{~m}$ (\#166). $a=b, \alpha=\beta=90^{\circ}, \gamma=120^{\circ}$, Wyckoff positions: $\mathrm{M}\left(0,0,1 / 6-z_{M} / 6\right), \mathrm{B}(0,0,1 / 3+\delta)$.

[28] MS2: 8 atoms, space group P63/mmc (\#194). $a=b$, $\alpha=\beta=90^{\circ}, \gamma=120^{\circ}$, Wyckoff positions: $\mathrm{M}$ (4f) $\left(1 / 3,2 / 3,1 / 4-z_{M} / 4\right)$, B1 $(2 b)(0,0,1 / 4)$, B2 $(2 d)$ $(1 / 3,2 / 3,3 / 4)$.

[29] MS3: 5 atoms, space group $\mathrm{P} \overline{6} \mathrm{~m} 2$ (\#187). $a=b$, $\alpha=\beta=90^{\circ}, \gamma=120^{\circ}$, Wyckoff positions: M1 $(2 g)$ $\left(0,0, z_{M}\right), \mathrm{M} 1$ (1d) $(1 / 3,2 / 3,1 / 2), \mathrm{B} 1$ (1c) $(1 / 3,2 / 3,0), \mathrm{B} 2$ (1e) $(2 / 3,1 / 3,0)$.

[30] Y. Zhu, L. Wu, V. Volkov, Q. Li, G. Gu, A.R. Moodenbaugh, M. Malac, M. Suenaga, and J. Tranquada, Physica C 356, 239 (2001).

[31] Y. Yan and M.M. Al-Jassim, Phys. Rev. B 66, 052502 (2002).

[32] $\delta: 3$ atoms in the primitive unit cell, space group $\mathrm{Cmmm}$ (\#50). $\alpha=\beta=\gamma=90^{\circ}$, Wyckoff positions: M (2c) $(1 / 2,0,1 / 2), \mathrm{B}(x, 0,0) . \delta-\mathrm{AuB}_{2}: a=5.762 \AA, b=2.721$ $\AA, c=4.328 \AA, x=0.3498$.

[33] S. Curtarolo, D. Morgan, and G. Ceder, Calphad 29, 163-211 (2005).

[34] S. Curtarolo, A. N. Kolmogorov, and F. H. Cocks, Calphad 29, 155-161 (2005).

[35] Li, Na, K, Rb, and Cs have nearly degenerate bcc and fcc energies at zero pressure.

[36] G. Mair, R. Nesper, and H.G. von Schnering, J. Solid State Chem. 75, 30 (1988).

[37] G. Mair, H.G. von Schnering, W. Wörle, and R. Nesper, Z. Anorg. Allg. Chem. 625, 1207 (1999).

[38] Z. Liu, X. Qu, B. Huang, and Z. Li, J. Alloys Compd. 311, 256 (2000).

[39] H. Rosner and W.E. Pickett, Phys. Rev. B 67, 054104 (2003).

[40] H.B. Borgstedt and C. Guminski, J. Phase Equilibria 24, 572 (2003).

[41] B. Albert, Eur. J. Inorg. Chem. 2000, 1679 (2000).

[42] F.E. Wang, M.A. Mitchell, R.A. Sutula, J.R. Holden, and L.H. Bennett, J. Less-Common Met. 61, 237 (1978).

[43] F.E. Wang, Metall. Trans. A 10, 343 (1979).

[44] S. Dallek, D.W. Ernst, and B.F. Larrick, J. Electrochem. Soc. 126, 866 (1979).

[45] According to our $a b$ initio results this structure has large positive formation energy $(0.5 \mathrm{eV} /$ atom $)$.

[46] M. Wörle and R. Nesper, Angew. Chem. Int. Ed. 39, 2349 (2000).

[47] A. N. Kolmogorov and V. H. Crespi, Phys. Rev. Lett. 85, 4727 (2000); T. Vuković, M. Damnjanović, I. Milos̃ević, Phisica E 16, 259-268 (2003).

[48] J.H. Xu, T. Oguchi, and A.J. Freeman, Phys. Rev. B 35, 6940 (1987); P. Ravindran, L. Fast, P.A. Korzhavyi, B. Johansson, J. Wills, and O. Eriksson, J. Appl. Phys. 84, 4891 (1998).

[49] The difference of the equilibrium boron-boron bond lengths in the $\mathrm{LiB}_{y}$ and $\mathrm{B}^{1 / y}$ phases caused by the constant stress from the lithium sublattice $\left(F_{L i^{+}}=0.76 \mathrm{eV} / \AA\right)$ is found as $a_{L i B_{y}}-a_{B^{-1 / y}}=-F_{L i+} /\left(\partial^{2} E_{B^{-1 / y}}(a) / \partial a^{2}\right)$, where $\partial^{2} E_{B^{-1 / y}}(a) / \partial a^{2}$ is $26.5 \mathrm{eV} / \AA^{2}$ at $\mathrm{y}=1$ and $22.8 \mathrm{eV} / \AA^{2}$ at $\mathrm{y}=0.8$.

[50] In fact, if we define $H^{*}$ as the intersection of the tangent and the $x=0.5$ line in Fig. $6\left(H^{*} \equiv-0.5 \times\right.$ tangent $)$, then $H^{*}$ is lower than $H_{M S 2-L i B}$ by approximately 40 $\mathrm{meV} /$ atom for all the pressures considered.

[51] M. Hanfland, K. Syassen, N. E. Christensen, and D. L. Novikov, Nature 408, 174 (2000).

[52] B. Albert, Angew. Chem. 110, 1135 (1998); B. Albert and K. Hofmann, Z. Anorg. Allg. Chem. 625, 709 (1999).

[53] R. Naslain and J. Etourneau, Compt. Rend. 263, 484 (1966).

[54] B. Albert, K. Hofmann, C. Fild, H. Eckert, M. Schleifer, and R. Gruehn, Chem.-Eur. J. 6, 2531 (2000).

[55] B. Aronsson, E. Stenberg, and J. Aselius, Acta Chem. Scand. 14, 733 (1960).

[56] H.B. Michaelson, J. Appl. Phys. 48, 4729 (1977).

[57] Work functions of a [111] monolayer of different metals are likely to follow the same order as those of bulk structure.

[58] $R_{M T}^{A g}=2.0$ a.u., $R_{M T}^{B}=1.6$ a.u., and $R_{M T} \cdot K_{\max }=8.0$.

[59] T. Sun, Y.G. Zhao, R. Fan, X.P. Zhang, B.G. Liu, Y.H. Xiong, and P.J. Li, J. Superconductivity 17, 473 (2004).

[60] A. Shukla, M. Calandra, M. d'Astuto, M. Lazzeri, F. Mauri, C. Bellin, M. Krisch, J. Karpinski, S. M. Kazakov, J. Jun, D. Daghero, and K. Parlinski, Phys. Rev. Lett. 90, 095506 (2003).

[61] M. Calandra, A.N. Kolmogorov, and S. Curtarolo, in preparation. 